\title{
Mixing and chemical reactions in a turbulent liquid mixing layer
}

\author{
By M. M. KOOCHESF A HANI AND P. E. DIMOTAKIS \\ Graduate Aeronautical Laboratories, California Institute of Technology, \\ Pasadena, CA 91125, USA
}

(Received 13 August 1985 and in revised form 11 February 1986)

An experimental investigation of entrainment and mixing in reacting and nonreacting turbulent mixing layers at large Schmidt number is presented. In nonreacting cases, a passive scalar is used to measure the probability density function (p.d.f.) of the composition field. Chemically reacting experiments employ a diffusionlimited acid-base reaction to directly measure the extent of molecular mixing. The measurements make use of laser-induced fluorescence diagnostics and high-speed, real-time digital image-acquisition techniques.

Our results show that the vortical structures in the mixing layer initially roll-up with a large excess of fluid from the high-speed stream entrapped in the cores. During the mixing transition, not only does the amount of mixed fluid increase, but its composition also changes. It is found that the range of compositions of the mixed fluid, above the mixing transition and also throughout the transition region, is essentially uniform across the entire transverse extent of the layer. Our measurements indicate that the probability of finding unmixed fluid in the centre of the layer, above the mixing transition, can be as high as 0.45 . In addition, the mean concentration of mixed fluid across the layer is found to be approximately constant at a value corresponding to the entrainment ratio. Comparisons with gas-phase data show that the normalized amount of chemical product formed in the liquid layer, at high Reynolds number, is $50 \%$ less than the corresponding quantity measured in the gas-phase case. We therefore conclude that Schmidt number plays a role in turbulent mixing of high-Reynolds-number flows.

\section{Introduction}

It is now generally recognized that entrainment and mixing processes in a turbulent plane mixing layer are dominated by the dynamics of large-scale vortical structures. Much of this knowledge has come from investigations in gas-phase flows both in non-reacting cases, such as those of Brown \& Roshko $(1971,1974)$, Fiedler (1974) and Konrad (1976), and also in chemically reacting flows (Ganji \& Sawyer 1980; Wallace 1981; Pitz \& Daily 1983; Mungal \& Dimotakis 1984). Studies of mixing in liquid layers, by contrast, are very few.

The distinction between gas and liquid, in the context of mixing, is due to their different molecular diffusivities. Gases are characterized by a high mass-diffusion coefficient or, in non-dimensional form, a Schmidt number of order unity. Schmidt number, $S c=\nu / D$, is the ratio of the diffusion coefficient of momentum to that of mass. A liquid has a low mass-diffusion coefficient and in the present case of water as the working fluid $S c \approx 600$.

Qualitative observations of transport and mixing were made in liquid mixing 
layers by Winant \& Browand (1974) and Dimotakis \& Brown (1976). Breidenthal (1981) made the first quantitative measurement of the extent of mixing and the total amount of chemical product. The asymmetry of the mixed-fluid composition distribution in the liquid mixing layer and its consequences were reported by Koochesfahani, Dimotakis \& Broadwell (1985). While these liquid-layer studies confirmed the important role of the large-structure dynamics, Breidenthal's work also suggested that the amount of chemical product in the liquid layer is less than that in the gas-phase case and that, therefore, the Schmidt number plays a role in turbulent mixing of high-Reynolds-number shear layers.

We should point out that most models of turbulent transport and mixing take no explicit account of molecular transport (Bilger 1980; Pope 1981; Kollmann \& Janicka 1982; Jones \& Whitelaw 1982) and are, therefore, incapable of addressing Schmidt-number effects. In these models molecular diffusion is neglected in comparison with turbulent diffusion and transport and mixing are modelled as separate processes. It should also be noted that the applicability of traditional descriptions of turbulent entrainment and mixing, which are based on the concepts of gradient transport and eddy diffusivity, to flows dominated by large-structure dynamies is in doubt. See, for example, Walker (1979), Sreenivasan, Tavoularis \& Corrsin (1981) and Koochesfahani et al. (1985).

A recent attempt to model mixing and chemical-reaction processes in a turbulent shear layer that does not employ the concept of gradient diffusion has been the model of Broadwell \& Briedenthal (1982). They propose the following idealization for the fluid states in the mixing layer: (i) unmixed, i.e. pure fluids from the free streams, (ii) a homogeneous mixture at the entrainment ratio, and (iii) strained laminar diffusion layers (flame sheets) between the free-stream fluids. Their model provides a formalism which can address the results for gases and liquids (i.e. fluids of differing Schmidt number) by a single theory. Similar ideas were also proposed by Effelsberg \& Peters (1983) in terms of a model for the probability density function (p.d.f.) of a passive scalar.

This paper contains a detailed quantitative study of entrainment and mixing in a liquid shear layer. Flows in the pre- and post-mixing transition regime and also during the mixing transition are represented. The mixing transition is a consequence of the introduction of small-scale, three-dimensional motions into the layer (Konrad 1976; Bernal et al. 1979; Breidenthal 1981; Bernal 1981) and is associated with a marked increase in the amount of mixed fluid (or chemical product) in the turbulent region. Two types of experiments are described. In the first type, the p.d.f. of the composition field is estimated over a range of Reynolds numbers covering the extent of the mixing transition. This is done using a passive scalar technique in a non-reacting layer and is the liquid counterpart of Konrad's (1976) experiment. Some of these results have been presented in Koochesfahani \& Dimotakis (1985). In the second type of experiment, the extent of molecular mixing is directly measured using an isothermal, constant-density acid-base chemical reaction. These measurements are the liquid counterpart to the gas-phase experiment of Mungal \& Dimotakis (1984).

\subsection{Passive scalar versus chemical reaction}

It is known that the passive scalar technique yields an upper bound to the actual molecular mixing (e.g. Breidenthal 1981). In using this technique, a passive scalar contaminant, such as dye, is premixed with one of the streams. The concentration of dye within a small sampling volume, determined by the spatial and temporal resolution characteristics of the measuring apparatus, is then recorded as a function 
of time. The difficulty arises if the sampling volume is larger than the smallest mixing scales, as is usually the case. Under these circumstances, it is impossible to determine whether the two fluids are mixed or not within the measurement resolution. Breidenthal points out that the problem of finite sampling volume can be solved by using a fast and irreversible chemical reaction of the type $A+B \rightarrow P$. If dilute reactant $A$ is added to one stream and $B$ to the other, then the amount of chemical product formed is equal to the amount of molecular-scale mixing between the two streams at the reaction stoichiometric ratio.

With all the drawbacks associated with the passive scalar method in determining the true extent of molecular mixing, it nevertheless is the simplest way to get an estimate of the composition field (i.e. the p.d.f.). While it is possible to determine the p.d.f. from chemical-reaction data with much better accuracy in representing molecular mixing (Wallace 1981), this would require many experiments at different reaction stoichiometric ratios. Using a passive scalar method, on the other hand, one obtains information about mixing at all mixture ratios in a single experiment. Chemical reaction measurements were also conducted, however, to assess the integrity of the conclusions reached on the basis of the passive scalar data and, of course, to also provide direct measurements of the amount of product.

The paper is organized as follows. Sections 2 and 3 give a brief description of the measuring techniques and instrumentation. Section 4 serves to introduce the definition and properties of quantities frequently used in this work and how they are related to the p.d.f. Discussion of results is presented in $\$ 5$ for the passive scalar measurements and in $\$ 6$ for chemical reaction data. Finally, comparisons with gaseous results are discussed in $§ 7$.

\section{Laser-induced fluorescence (LIF)}

The laser-induced fluorescence (LIF) technique has been used here in liquid-phase flows in two different ways. In non-reacting applications, a fluorescent dye is premixed with the low-speed free-stream fluid which is subsequently diluted in the shear layer as a result of mixing with the fluid from the high-speed free stream. No chemistry is involved and the dye acts as a conserved passive scalar. Experiments of this type are referred to here as passive scalar or dilution experiments. Recording the fluorescence intensity allows a quantitative measurement of the dye concentration and, therefore, the relative concentration of high-speed to low-speed fluid in the layer (Koochesfahani \& Dimotakis 1985). This is done by noting that the local instantaneous dye concentration $C_{\mathrm{d}}$ in a sampling volume is given by

$$
C_{\mathrm{d}}=C_{\mathrm{d}_{0}} \frac{v_{\mathbf{2}}}{v_{1}+v_{2}},
$$

where $C_{\mathrm{d}_{0}}$ is the free-stream dye concentration carried by the fluid on the low-speed side and $v_{1}$ and $v_{2}$ are the volumes of fluid from the high-speed and low-speed streams, respectively, in the sampling volume. The normalized concentration of high-speed fluid $\xi$ is thus equal to

$$
\xi=1-C_{\mathrm{d}} / C_{\mathrm{d}_{0}},
$$

where $\xi=v_{1} /\left(v_{1}+v_{2}\right)$ is defined as the high-speed-fluid volume fraction.

Chemically reacting cases make use of dyes whose fluorescence can be suppressed or induced by a particular chemical environment. The water-soluble organic dye disodium fluorescein (uranin), whose fluorescence is $\mathrm{pH}$ sensitive, has often been used 


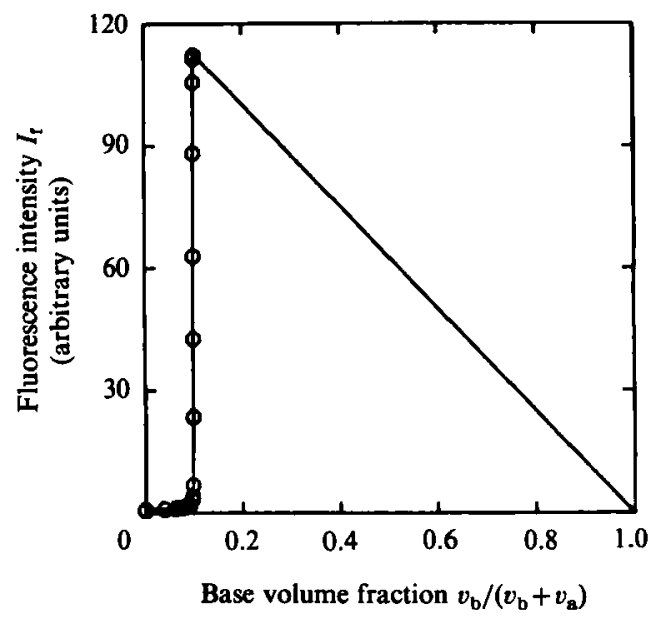

Figure 1. Fluorescence intensity versus base volume fraction.

in our laboratory. The chemical reaction consists of an acid-base reaction with the fluorescent dye premixed with the acid solution. The dye fluoresces efficiently, when excited by an argon-ion laser, provided that it is in a chemical environment whose $\mathrm{pH}$ is above a certain threshold. Generally, the fluorescence is off (suppressed) when the $\mathrm{pH}$ of the solution is below approximately 4 . The volume of base $v_{\mathrm{b}}$ required to titrate a unit volume $v_{\mathrm{a}}$ of the acid/dye solution and cross the $\mathrm{pH}$ threshold for fluorescence can be adjusted by the proper choice of the acid and base concentrations. Figure 1 illustrates the measured fluorescence intensity from the titration of a fixed volume of acid/dye solution. As we start adding the basic solution to the acid/dye solution, the fluorescence intensity is close to zero since the solution is very acidic. With the addition of more base, a point is reached where the $\mathrm{pH}$ threshold is crossed and the fluorescence 'turns on'. Beyond this mixture ratio, which in the present experiments was set to $v_{\mathrm{b}} / v_{\mathrm{a}}=1 / 10$, addition of more base merely dilutes the dye. Now the fluorescence intensity is only proportional to the dye concentration and hence proportional to the acid volume fraction. As can be seen, the fluorescence on/off transition can be made very sharp. This transition is reversible in the sense that the fluorescence intensity can be switched on and off by adding sufficient amounts of base or acid required to cross the $\mathrm{pH}$ threshold. Note that since the fluorescence of this dye depends on its local chemical environment, mixing can be monitored down to the molecular scale.

In chemically reacting LIF experiments, the fluorescence intensity is directly proportional to the product concentration, where chemical product is defined here as the molecularly (dye-bearing) mixed fluid whose local $\mathrm{pH}$ is above the fluorescence threshold. With this operational definition, the local instantaneous product concentration $C_{\mathrm{p}}$, normalized with its maximum possible value $\left(C_{\mathrm{p}}\right)_{\max }$ which is reached at the threshold conditions for fluorescence 'turn-on', is given by

$$
\frac{C_{\mathrm{p}}}{\left(C_{\mathrm{p}}\right)_{\max }}=\frac{I_{\mathrm{f}}}{\left(I_{\mathrm{f}}\right)_{\max }} .
$$

$I_{\mathrm{f}}$ is the measured fluorescence intensity and $\left(I_{\mathrm{f}}\right)_{\max }$ its maximum possible value reached at the fluorescence threshold conditions (see figure 1 ).

It should be mentioned that defining the product concentration in terms of the 


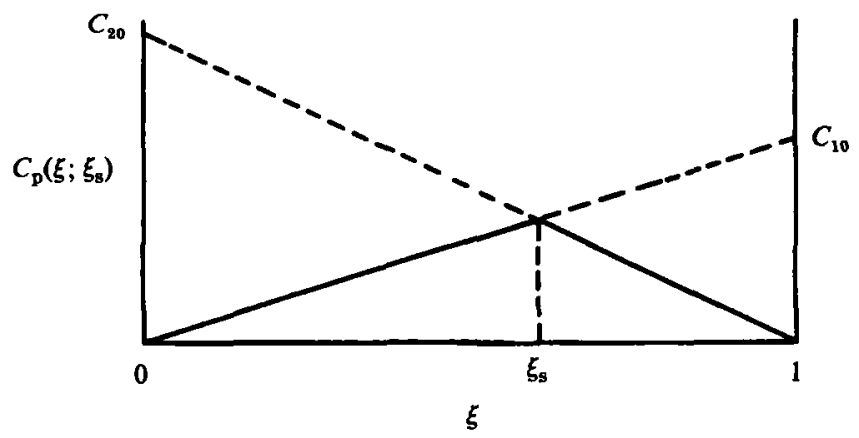

Figure 2. $C_{\mathrm{p}}$ versus $\xi$ in fast irreversible reactions.

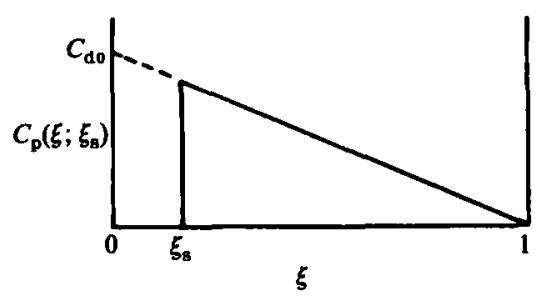

(a)

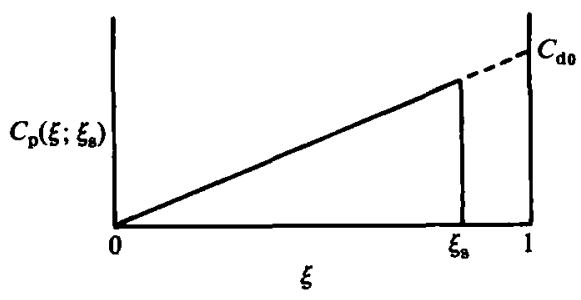

(b)

Figure 3. $C_{\mathrm{p}}$ versus $\xi$ in chemically reacting LIF experiments. (a) Base on high-speed side and acid on low-speed side, (b) Base on low-speed side and acid on high-speed side.

fluorescence intensity is not entirely arbitrary and is connected with the structure of the product concentration in fast irreversible chemical reactions which we describe below. For simplicity, consider a one-step reaction of the type $A+B \rightarrow P$ with unity molar stoichiometric coefficients. Suppose that the dilute reactants $A$ and $B$ are carried in the high-speed and low-speed streams at free-stream concentrations of $C_{10}$ and $C_{20}$ respectively. If the reaction is infinitely fast and irreversible, the product concentration will be dictated by the reactant in short supply and is given by (e.g. Bilger 1980)

$$
C_{\mathrm{p}}\left(\xi ; \xi_{\mathrm{s}}\right)= \begin{cases}C_{10} \xi & \text { for } \xi<\xi_{\mathrm{s}}, \\ C_{20}(1-\xi) & \text { for } \xi>\xi_{\mathrm{s}}\end{cases}
$$

where $\xi_{\mathrm{s}}=C_{20} /\left(C_{10}+C_{20}\right)$ is the stoichiometric mixture fraction. A sketch of $C_{\mathrm{p}}\left(\xi ; \xi_{\mathrm{s}}\right)$ is shown in figure 2. For LIF experiments two cases can be defined, depending on which chemical is on the high-speed side, as follows:

(a) base on the high-speed side, acid/dye on the low-speed side

$$
C_{\mathrm{p}}\left(\xi ; \xi_{\mathrm{s}}\right)= \begin{cases}0 & \text { for } \xi<\xi_{\mathrm{s}}, \\ C_{\mathrm{d}_{0}}(1-\xi) & \text { for } \xi>\xi_{\mathrm{s}}\end{cases}
$$

where $\xi_{\mathrm{s}}=\left[v_{\mathrm{b}} /\left(v_{\mathrm{a}}+v_{\mathrm{b}}\right)\right]_{\mathrm{thr}}$ is the base volume fraction at the threshold condition for fluorescence 'turn on' and $C_{\mathrm{d}_{0}}$ is the free-stream dye concentration carried by the acid solution.

(b) base on the low-speed side, acid/dye on the high-speed side

$$
C_{\mathrm{p}}\left(\xi ; \xi_{\mathrm{s}}\right)= \begin{cases}C_{\mathrm{d}_{0}} \xi & \text { for } \xi<\xi_{\mathrm{s}} \\ 0 & \text { for } \xi>\xi_{\mathrm{s}}\end{cases}
$$




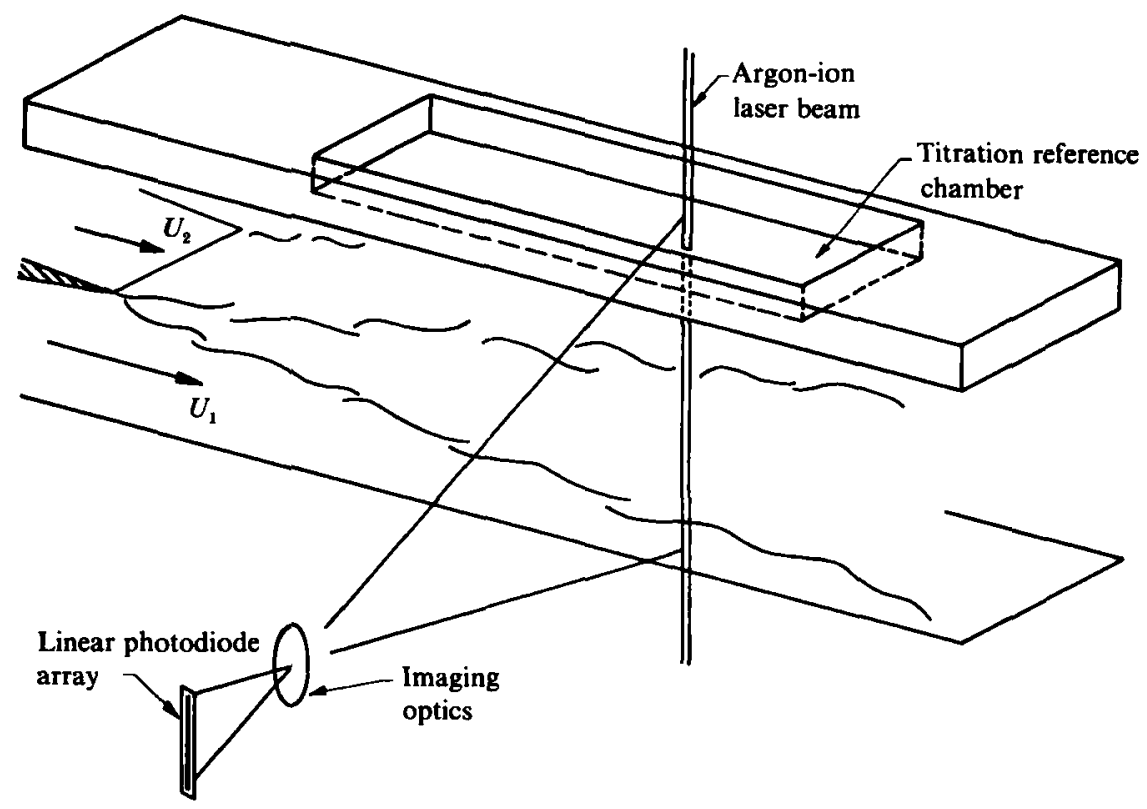

Figure 4. Optical set-up.

where $\xi_{\mathrm{s}}=1-\left[v_{\mathrm{b}} /\left(v_{\mathrm{a}}+v_{\mathrm{b}}\right)\right]_{\mathrm{thr}}$. A sketch of $C_{\mathrm{p}}$ for these two cases is shown in figure 3 . Note that the only distinguishing feature between cases $(a)$ and $(b)$ is the exchange of the chemical composition of the free streams between the two sets ('flip' experiments), see also Koochesfahani et al. (1985) and Mungal \& Dimotakis (1984).

Comparison of figures 2 and 3 , or equations (4) and $(5 a, b)$, indicates that the product concentrations are identical in form with one exception. The product concentration in the LIF experiments is a 'one-sided' triangle. This, of course, is the consequence of the chemical-environment requirements for fluorescence. Another difference, the reversibility of the reaction, is discussed in $\$ 7$ where the amount of product in gas- and liquid-phase layers are compared.

\section{Experimental facility and instrumentation}

\subsection{Shear-layer apparatus}

The experiments were conducted in the water mixing-layer facility designed by Breidenthal (1981). It is a gravity-driven-flow apparatus in which the free-stream fluids are supplied from two independent reservoirs. In the dilution (passive scalar) experiments, the low-speed free-stream fluid was prepared by adding sodium fluorescein dye to water to a concentration of about $5 \times 10^{-7} \mathrm{M}$ (molar concentration), whereas the high-speed free stream was pure water.

In reacting experiments the chemical reaction between sulfuric acid $\left(\mathrm{H}_{2} \mathrm{SO}_{4}\right)$ and sodium hydroxide $(\mathrm{NaOH})$ was utilized with the fluorescein dye premixed with the acid solution. The highest dye concentration used was $10^{-5} \mathrm{M}$. The acid and base concentrations that were used resulted in the titration curve of figure 1 . The facility was modified to include a titration reference chamber, shown in figure 4, which contained, for each run, the titrated acid-base solution corresponding to the maximum possible product concentration for that run. Densities of the two free-stream fluids were matched, when necessary, by adding sufficient amounts of sodium fulfate 


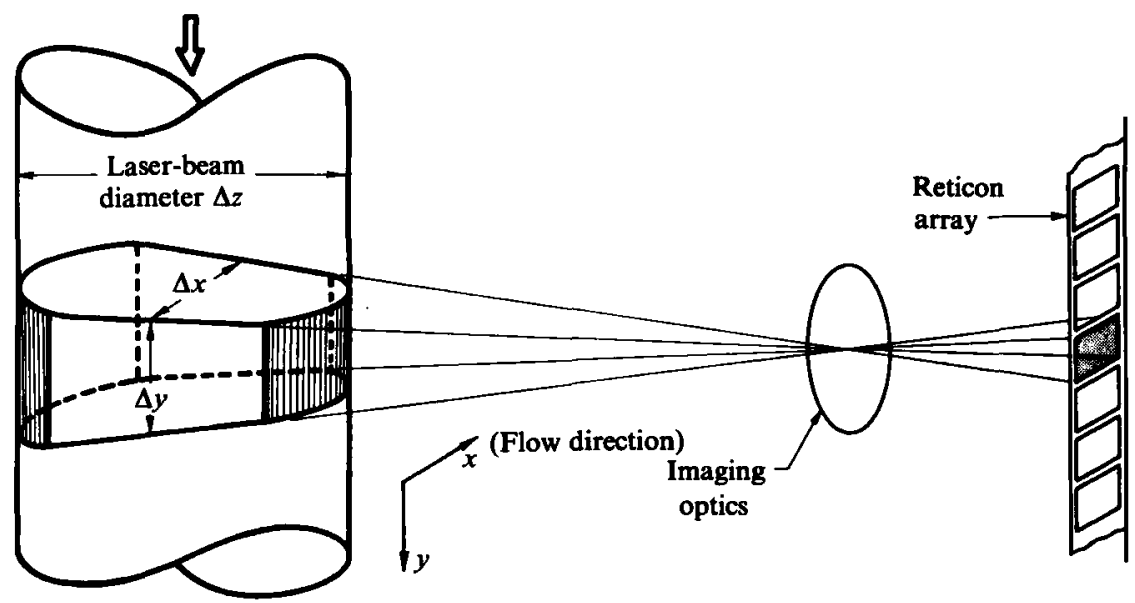

Figure 5. Schematic of focusing arrangement.

$\left(\mathrm{Na}_{2} \mathrm{SO}_{4}\right)$ salt to the acid/dye solution. In all cases the resulting Richardson number was at least two orders of magnitude smaller than the value below which, according to Koop \& Browand (1979), buoyancy effects could safely be neglected. The Richardson number, in this case, is defined by

$$
R i=\frac{\Delta \rho g \delta}{\bar{\rho}(\Delta U)^{2}}
$$

where $\Delta \rho=\rho_{1}-\rho_{2}$ is the density difference across the layer, $\bar{\rho}=\frac{1}{2}\left(\rho_{1}+\rho_{2}\right)$ the mean density, $g$ the gravitational constant, $\delta$ the vorticity thickness, and $\Delta U=U_{1}-U_{2}$ the velocity difference across the layer.

\subsection{Optical set-up}

The beam of a $3 \mathrm{~W}$ argon-ion laser was collimated by a converging lens to produce a thin beam (diameter in the range $0.5-1 \mathrm{~mm}$ ) aligned with the transverse direction of the mixing layer at the mid-span of the test section. Variations of the beam diameter along the width of the text section were minimized by using a long focal-length lens. The fluorescence intensity along the beam was measured by a Reticon self-scanning linear photodiode array (see figure 4). In the dilution experiments a 512-element array was used and the laser (Coherent Radiation CR-3) was operated in the multi-line mode. For reacting experiments, the photodiode array had 1024 elements and the single-line $514.5 \mathrm{~nm}$ of the laser (Lexel model 95) was used.

Each element (pixel) of the array receives light from an area in the object plane that is determined by the effective aperture of the pixel $(26 \mu \mathrm{m} \times 25 \mu \mathrm{m})$ and the magnification ratio of the collecting optics (typically $1: 4$ ). This area defines the spatial resolution in the plane of the array and for these measurements was given by $\Delta x \times \Delta y \approx 104 \mu \mathrm{m} \times 100 \mu \mathrm{m}$, where $\Delta x$ and $\Delta y$ are the spatial resolution in the flow direction $x$ and the transverse direction $y$ respectively. The spanwise resolution $\Delta z$ is determined by the laser-beam diameter, as indicated in figure 5 . It should be mentioned that these resolution characteristics apply only to static conditions. The actual dynamic resolution in a particular direction is the sum of the static resolution and the distance the flow moves in that direction during the array scan time. See Koochesfahani (1984) for further details. 


\subsection{Data acquisition}

Reticon array data were acquired by a computer data-acquisition system based on a PDP-11/23 CPU. The output of the array was digitized by a single channel 8-bit A/D system capable of $10^{7}$ conversions/s. For the dilution experiments, the array was clocked at $640 \mathrm{kHz}$, which corresponded to a scan time (or temporal resolution) of $0.8 \mathrm{~ms}$, and a scan rate of $1250 \mathrm{scans} / \mathrm{s}$. In one case, data were recorded also at $8 \mathrm{~ms}$ scan time ( $64 \mathrm{kHz}$ clock) in order to investigate the effects of degrading the temporal resolution. In the reacting experiments the array clocking rate was $512 \mathrm{kHz}$ corresponding to a scan time of $2 \mathrm{~ms}$ or a scan rate of $500 \mathrm{scans} / \mathrm{s}$.

Two types of data were generally recorded. The first type was acquired at the array clocking rate, stored in the computer memory at that rate, and subsequently recorded on a high-speed disc. The amount of data, in this case, as limited by the system memory, corresponded to the passage of only a small number of large structures. This type of data, though not useful for reliable statistics, was suitable for examining the detailed structure of the flow.

A second set of data was obtained for long time averages and statistical purposes by acquiring a long contiguous data record. Sharing the computer bus cycles between the $\mathrm{A} / \mathrm{D}$ board and the disc controller, however, limited the fastest rate at which large amounts of data could be digitized and transferred to the disc continuously. To accommodate the maximum data rate, only 1 out of every 8 pixels in the dilution runs (and 1 out of 4 in reacting cases) were digitized. This, in effect, reduced the transfer rate to a manageable value without compromising the spatial and temporal resolution of each pixel. With this arrangement, large amounts of data (up to the disc capacity) could be obtained continuously at 64 points (dilution runs) or 256 points (reacting runs) across the layer, essentially simultaneously. The sustained data rate in the reacting case was $128 \mathrm{kbyte} / \mathrm{s}$, roughly half the capability of the acquisition system. The amount of data per run typically corresponded to the passage of about 100 large structures (in excess of $2.5 \times 10^{6}$ numbers).

\subsection{Data reduction}

The steps for computing the local instantaneous dye concentration from the raw fluorescence-intensity data are described in Koochesfahani \& Dimotakis (1985). The main result, for this discussion, is that the output voltage of a given pixel below saturation is linearly proportional to the fluorescence intensity issued from the corresponding imaged point. The fluorescence intensity, in turn, is linearly proportional to the local dye concentration and the local laser intensity. Therefore, the pixel output is linear in the local dye concentration. The effects of non-uniformity of imaging and pixel sensitivity, laser-beam diameter variation and possible small misalignments between the array and the beam are removed by calibrating the system with a dilute uniform dye solution in the test section. The laser-beam attenuation due to absorption by the dye is also removed on the scan-by-scan basis. Note that the ability to remove attenuation effects is a unique advantage of linear photodiode arrays which provide information at many closely spaced points essentially simultaneously permitting the attenuation line integral to be computed for each sean.

In the dilution experiments, the high-speed-fluid concentration is computed according to (2). Since the full transverse extent of the shear layer is imaged on the array, the reference free-stream dye concentration $C_{\mathrm{d}_{0}}$ is available in each scan making the calculation of $(2)$ relatively simple. The product concentration in reacting experiments is obtained using (3). The reference value $\left(I_{\mathrm{f}}\right)_{\max }$ is available in each scan in the titration reference chamber (figure 4) which is also imaged on the array. 


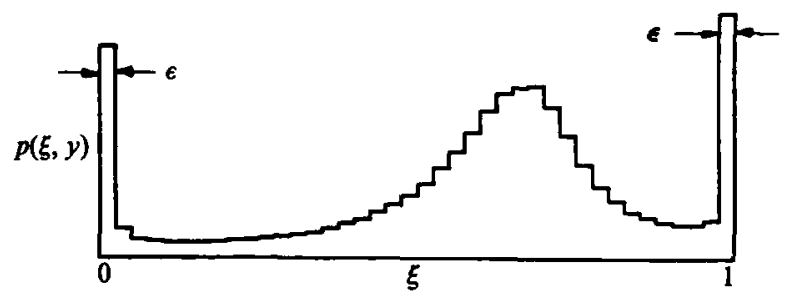

Figure 6. A typical p.d.f.

\section{Probability density function (P.D.F.)}

This section describes the definitions and properties of the quantities discussed in this paper, and how they are related to the p.d.f. of the high-speed-fluid concentration $\xi$ measured in the dilution experiments.

We denote the p.d.f. of $\xi$ at a given point $y$ in the layer by $p(\xi, y)$. Normalization of the p.d.f. requires that

$$
\int_{0}^{1} p(\xi, y) \mathrm{d} \xi=1
$$

The average concentration of high-speed fluid $\bar{\xi}$ is then given by

$$
\bar{\xi}(y)=\int_{0}^{1} p(\xi, y) \mathrm{d} \xi
$$

A typical $p(\xi, y)$ is shown in figure 6. The two 'delta' functions at $\xi=0,1$ are associated with the pure unmixed fluids of the low-and high-speed sides respectively. Their widths are dictated by the overall signal-to-noise ratio of the measurement and, therefore, are not zero in practice. In these measurements, the range of $\xi$ was divided into 32 levels so that the value of $\epsilon$ was 0.031 . Concentrations in the range $0 \leqslant \xi<\epsilon$ are assigned to pure (unmixed) low-speed fluid and $1-\epsilon<\xi \leqslant 1$ to pure high-speed fluid. The range $\epsilon \leqslant \xi \leqslant 1-\epsilon$ would, therefore, correspond to mixed fluid. With this description, the probabilities $P_{0}$ and $P_{1}$ of finding unmixed fluids from lowand high-speed sides respectively, are given by

$$
P_{0}(y)=\int_{0}^{\epsilon} p(\xi, y) \mathrm{d} \xi, \quad P_{1}(y)=\int_{1-\epsilon}^{1} p(\xi, y) \mathrm{d} \xi .
$$

We can characterize the mixed fluid p.d.f. (i.e. excluding the two delta functions) by its area and first moment. The area under the mixed fluid p.d.f. $P_{\mathrm{m}}$ gives the total probability of finding mixed fluid at any concentration. It is given by

$$
P_{\mathrm{m}}(y)=\int_{\epsilon}^{1-\epsilon} p(\xi, y) \mathrm{d} \xi
$$

A value of $P_{\mathrm{m}}(y)$ of less than unity signifies the presence of unmixed fluid. The reference location $y=0$ which is used throughout this work was arbitrarily chosen to coincide with the point at maximum $P_{\mathrm{m}}(y)$. Additionally, a reference thickness $\delta_{1}$ was defined as the $1 \%$ width of the total mixed-fluid probability $P_{\mathrm{m}}(y)$ where $P_{\mathrm{m}}$ had dropped to $1 \%$ of its maximum value. Since this width was found to agree very well with the visual width $\delta_{\text {vis }}$ of the layer (Koochesfahani 1984), $\delta_{1}$ and $\delta_{\text {vis }}$ are used interchangeably here. 
The first moment of $p(\xi, y)$, also called the average mixed-fluid concentration, is given by

$$
\xi_{\mathrm{m}}(y)=\frac{\int_{\varepsilon}^{1-\epsilon} \xi p(\xi, y) \mathrm{d} \xi}{\int_{\epsilon}^{1-\epsilon} p(\xi, y) \mathrm{d} \xi}=\frac{\int_{\epsilon}^{1-\epsilon} \xi p(\xi, y) \mathrm{d} \xi}{P_{\mathrm{m}}(y)}
$$

Finally, we define the total mixed-fluid concentration for the entire layer by

$$
\xi_{\mathrm{M}}=\frac{\int_{-\infty}^{+\infty} P_{\mathrm{m}}(y) \xi_{\mathrm{m}}(y) \mathrm{d} y}{\int_{-\infty}^{+\infty} P_{\mathrm{m}}(y) \mathrm{d} y}
$$

\section{Passive scalar (dilution) results}

Data are presented here over a range of Reynolds numbers covering the extent of the mixing transition. Breidenthal's (1981) data were used to determine the flow parameters required to generate pre- and post-mixing-transition flows. For the cases studied here, the mixing transition starts at a local Reynolds number of approximately 5600 and is completed by a Reynolds number of roughly 17000 (e.g. see figure 13). The local Reynolds number is defined here by $R e=\delta_{1} \Delta U / \nu$ and is based on the local thickness $\delta_{1}$ and the velocity difference across the layer $\Delta U$.

\subsection{Results below the mixing transition}

Typical digital LIF data for this case are displayed in figure 7 (plate 1). The two photographs of a digital image-processing monitor screen, show the concentration field at a downstream distance from the splitter plate of $x=18 \mathrm{~cm}$ with $U_{1}=13 \mathrm{~cm} / \mathrm{s}$ and velocity ratio $r=U_{2} / U_{1}=0.46$. The local Reynolds number is $R e \approx 1750$. Each picture represents 384 successive scans, each consisting of 400 points across the layer, for a total flow time of $307 \mathrm{~ms}$.

It should be emphasized that, technically speaking, these are not 'photographs'. The vertical extent maps onto $40 \mathrm{~mm}$ of the transverse coordinate of the shear layer. The horizontal extent is time (increasing from right to left). We have used pseudocolour assignment to various concentration levels in order to facilitate the interpretation of the composition field in these $(y, t)$-pictures. The colour assignment is such that red corresponds to pure low-speed fluid $(\xi=0)$, blue to pure high-speed fluid $(\xi=1)$, and the rest of the colour spectrum indicates mixed fluid (i.e. $0<\xi<1$ ).

Figure 7 shows that the concentration field before the mixing transition is rather simple. A given point in the layer spends most of its time in alternating regions of pure unmixed high-and low-speed fluids. There is very little mixing and that occurs at the thin interfaces separating the two fluids. It is clear that if this shear layer were carrying reactants in its free streams, the amount of chemical product would be very small, in agreement with the measurements of Briedenthal (1981).

An interesting point is the large excess of high-speed fluid found in the core of the structures. As we shall see later, this has implications for how the mixed-fluid composition evolves during the mixing transition. It must be emphasized that this initial large asymmetry in the core composition is different from and is not due to the entrainment into the fully developed layer, which is also asymmetric. We have no clear explanation for the asymmetric roll-up behaviour at this point. 


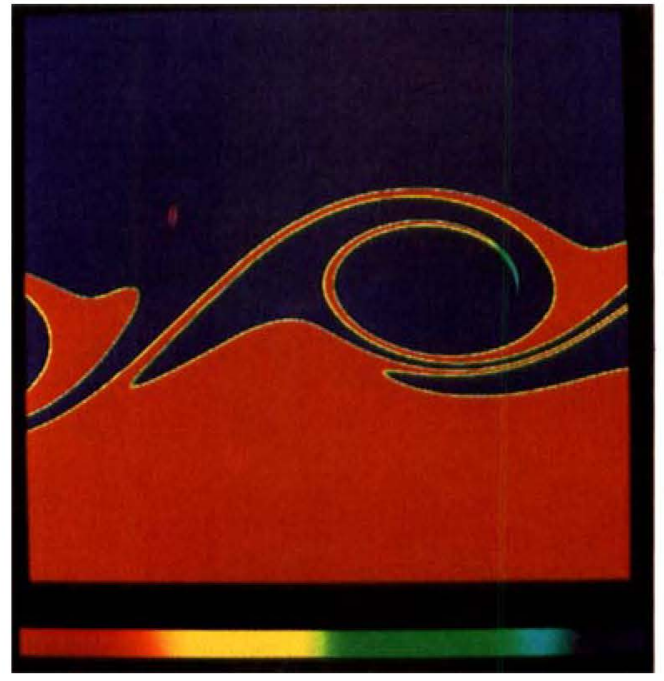

(a)

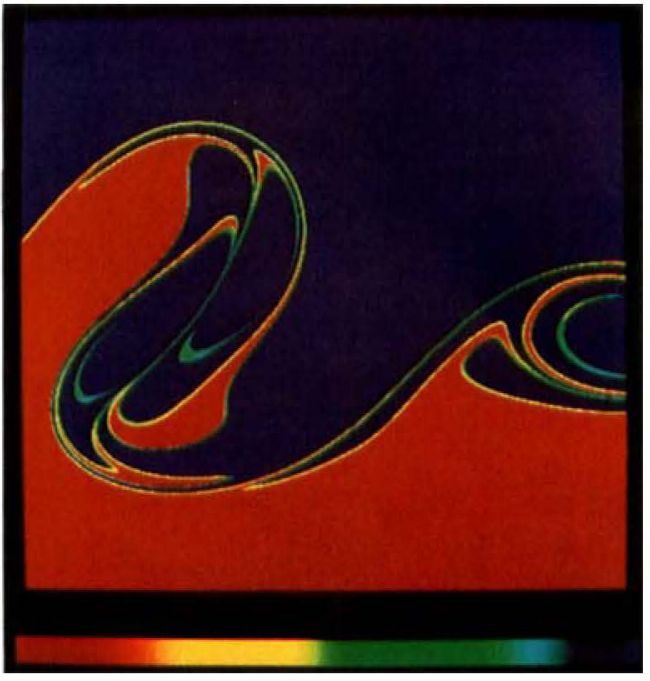

(b)

FIGURE 7. Digital LIF picture below the mixing transition, $r=0.46, R e=1750$. (a) single vortex, (b) pairing vortices.

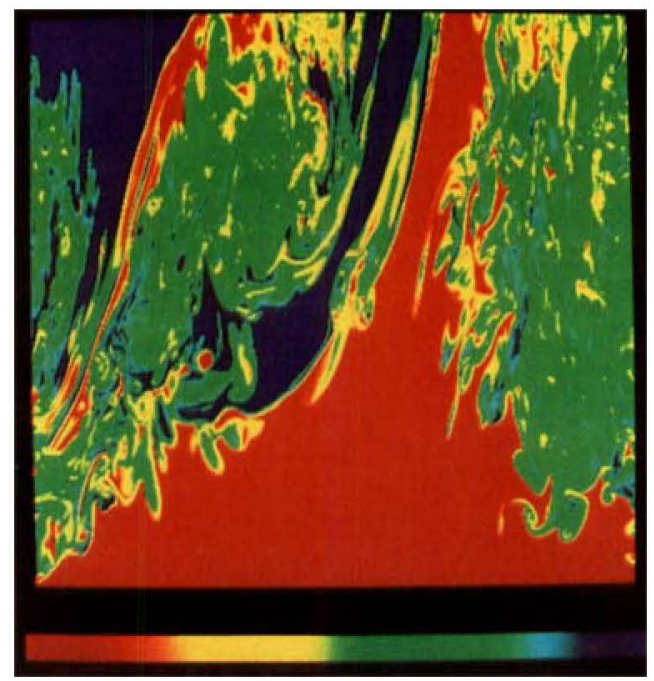

Figure 9. Digital LIF picture above the mixing transition, $r=0.38, R e=23000$. 


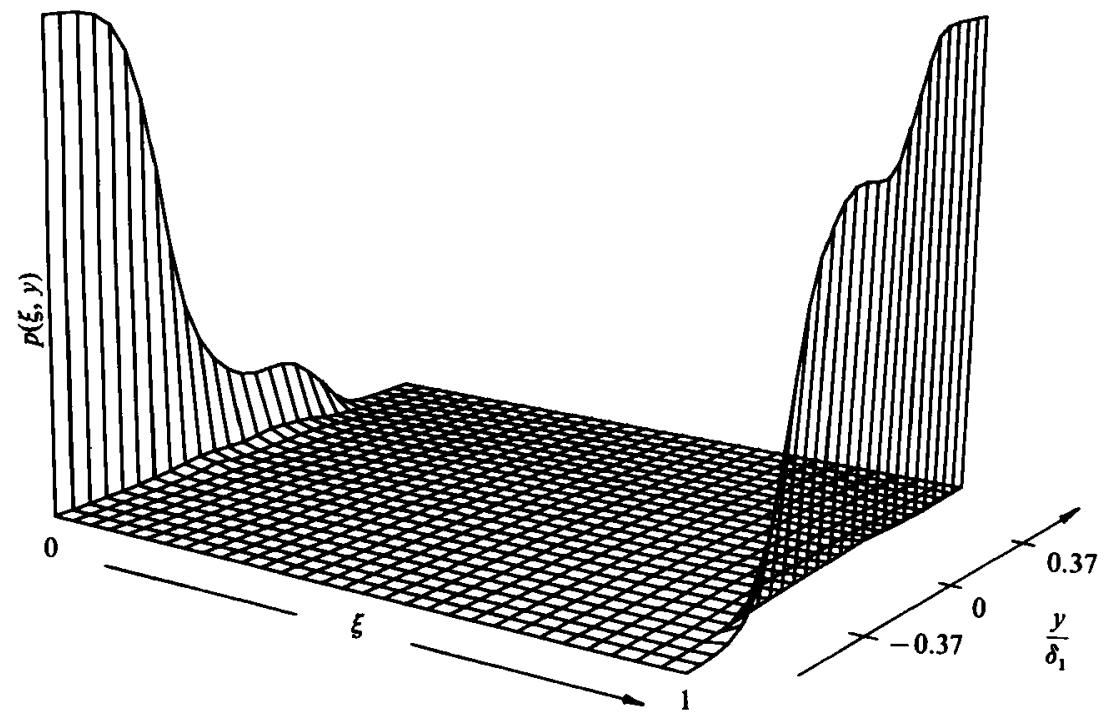

Figure 8. P.D.F. of the composition field below the mixing transition, $r=0.38, R e \approx 28000$.

To conclude this section, a typical p.d.f. of the composition field across the entire layer below the mixing transition is presented in figure 8 . The data for this figure were obtained at $x=7 \mathrm{~cm}$ with $U_{1}=30 \mathrm{~cm} / \mathrm{s}$ and $r=0.38$. The local Reynolds number was 2800 . Consistent with the results already discussed, the p.d.f.'s in this case are characterized by two delta functions located at $\xi=0$ and 1 , the heights of which depend on the location in the layer, with practically no mixed fluid in between. The excess of high-speed fluid in the cores reveals itself in the form of a distinct 'hump' in the probability of finding pure high-speed fluid around the middle of the layer. As a result of this, there is also a corresponding depression in the probability of finding pure low-speed fluid.

\subsection{Results above the mixing transition}

The data presented for this case were recorded at $x=25 \mathrm{~cm}$ with $U_{1}=70 \mathrm{~cm} / \mathrm{s}$ and $r=0.38$. The resulting local Reynolds number was $R e \approx 23000$. A $(y, t)$ 'photograph' of the composition field was prepared in the same fashion as figure 7 and is shown in figure 9 (plate 1). The change in the apparent aspect ratio of the large structures is the result of the fixed scanning rate that was used in these measurements and the increase of the free-stream velocities. The p.d.f. of the concentration field across the entire layer of this case appears in figure 10. It should be noted that this is the p.d.f. of a long contiguous data set corresponding to the passage of approximately 100 large structures.

Two main features are apparent in figures 9 and 10. The first is that the composition distribution of the mixed fluid is essentially uniform across the entire transverse extent of the mixing layer; predominant values of mixed-fluid concentration are the same regardless of the position in the layer. This is the reason for the rather uniform colour in the core of the vortical structure in figure 9 , which is more or less all green. The colour has no tendency toward blue as the high-speed steam is reached or towards red on the low-speed side.

The second feature is that the mixed-fluid composition is asymmetric and biased toward the high-speed fluid. The average concentration of mixed fluid for the entire layer $\xi_{M}$ was estimated (using (11)) to be equal to 0.60 indicating a mixture of 1.5 


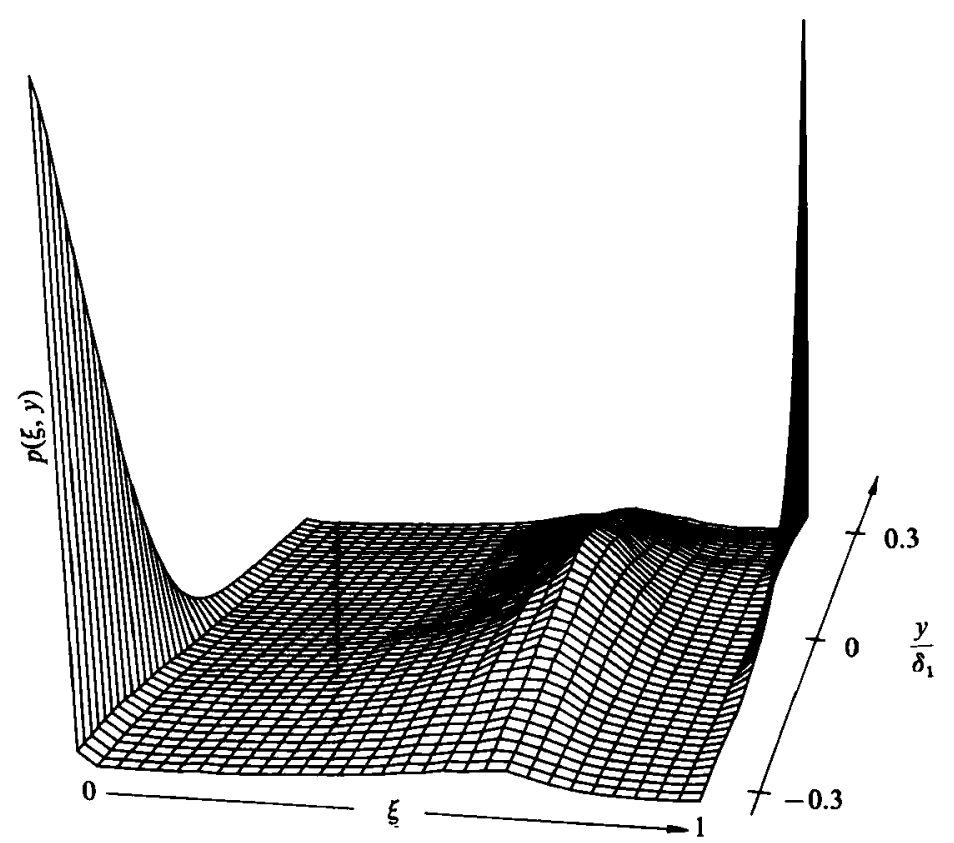

FIGURE 10. P.D.F. of the composition field above the mixing transition, $r=0.38, R e \approx 23000$.

parts of high-speed to 1 part of low-speed fluid. If we accept that the overall mean composition of the mixed fluid in the layer is determined by the amounts entrained from the two sides, then we would conclude that the entrainment ratio $E$ is 1.5 . This value is somewhat higher than the value of 1.3 suggested by Konrad (1976) and Dimotakis (1984) for a shear layer at a velocity ratio of 0.38 . As will be made clearer later, it is generally very difficult to obtain the correct value of $\xi_{M}$ from dilution data. The main problem is the lack of adequate resolution of the measuring device which, in this case, tends to overestimate the value of $\xi_{M}$.

\subsection{The effect of resolution}

A fundamental problem associated with the passive scalar technique is that it cannot provide information on mixing within a scale smaller than the resolution volume connected with the measurement apparatus. As mentioned in $\$ 1.1$, the issue of resolution becomes important if the resolution volume is larger than the smallest mixing (diffusion) scales. For example, in the high-Reynolds-number experiment of the previous section, the smallest diffusion scale (Batchelor 1959 scale, estimated from $\lambda \approx S c^{-\frac{1}{2}} \delta_{1} R e^{-\frac{3}{4}}$ ) is about $1 \mu \mathrm{m}$, almost three orders of magnitude smaller than the resolution of the measurement. Under these circumstances, it is impossible to determine whether the two fluids are mixed or not within the resolution scale of the measurement device. This difficulty, in turn, results in the contamination of the measured p.d.f.'s. It should be noted, however, that the average concentration is measured with no ambiguity.

Figure 11. Effect of resolution on the transverse profiles. (a) Average high-speed fluid concentration: O, $0.8 \mathrm{~ms}$ scan; $\square, 8.0 \mathrm{~ms}$ scan. (b) Total mixed-fluid probability, symbols same as in (a). (c) Pure low-speed fluid: $\triangle, 0.8 \mathrm{~ms}$ scan; $\diamond, 8.0 \mathrm{~ms}$ scan; and pure high-speed fluid; $0,0.8 \mathrm{~ms}$ scan; $\square$, $8.0 \mathrm{~ms}$ scan. $(d)$ Average mixed-fluid concentration, see $(a)$ for symbols. 

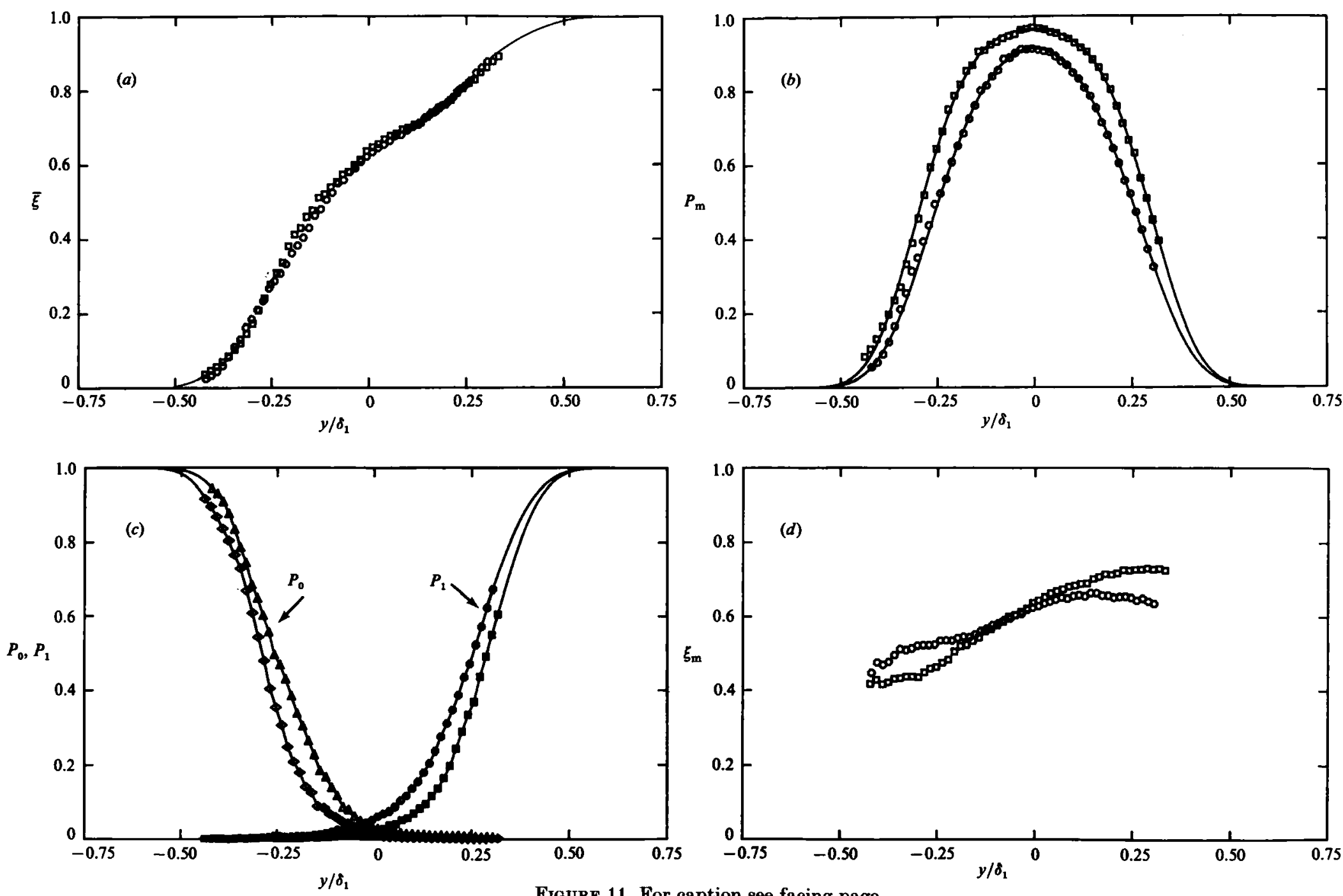

:

Frovre 11. For caption see facing page. 
The consequences of the lack of adequate resolution were discussed by Koochesfahani \& Dimotakis (1985). They considered the effect of a tenfold degradation of the temporal resolution (i.e. scan time was changed from 0.8 to $8 \mathrm{~ms}$ ) on the measured results. The findings, appearing in figures $11(a-d)$, for the flow described in $\$ 5.2$ were as follows. The average concentration $\bar{\xi}$ (figure $11 a$ ) is not affected since it is based on the correct measurement of average dye concentration not its fluctuations. The total probability of finding mixed fluid $P_{m}(y)$ in figure $11(b)$ increases everywhere in the layer upon lowering the resolution, indicating more mixed fluid in the layer. This is accommodated by decreases in the probabilities of finding unmixed fluid from lowand high-speed streams, $P_{0}$ and $P_{1}$ respectively, as shown in figure $11(c)$. It was found that the variation of the average mixed-fluid concentration $\xi_{\mathrm{m}}$ across the layer increases upon degrading the resolution (figure $11 d$ ). It is interesting to note that the lack of resolution seems to have changed the measured $\xi_{\mathrm{m}}$ profile in a direction that is qualitatively similar to what might be expected from an enhanced moleculardiffusion process. And finally the total mixed-fluid concentration $\xi_{\mathrm{M}}$ was calculated to be 0.62 , higher than the previous value of 0.60 .

Figure $11(b)$ shows that there is an estimated probability of a least $10 \%$ of finding unmixed fluid in the centre of the layer, and that the actual value may be even higher, if the resolution were to improve. The small variation of the $\xi_{m}$ profile across the layer, for the $0.8 \mathrm{~ms} \mathrm{scan}$, in figure $11(d)$ reflects the transverse uniformity of the mixed-fluid composition already discussed in conjunction with figure 10. An interesting possibility is that if the trend in figure $11(d)$ continues, the actual $\xi_{\mathrm{m}}$ profile may be even more uniform. It is not clear how measured results at two different resolution levels can be extrapolated to the limit of 'perfect' resolution. Such an extrapolation would in principle depend on the spectral characteristics of the composition fluctuations, which are not known a priori. It is possible, however, to get a direct estimate of all the quantities in figure 11 in the limit of 'perfect' resolution using chemical-reaction techniques. This will be described in $§ 6$.

\subsection{Evolution of the p.d.f. during the mixing transition}

Breidenthal (1981) has shown that the extent of mixing in a liquid shear layer during the mixing transition increases from a very low value before the transition to a higher value above the transition. His study, however, was concerned with the total amount of mixing, and the actual distribution of the mixed-fluid concentration in the transition region was not known. In this section, we present data that describe the evolution of the p.d.f.'s from the structure shown in figure 8 into that of figure 10 in the course of the mixing transition. Two sets of data in the transition region were recorded at $x=17 \mathrm{~cm}$ and a velocity ratio $r=0.38$. For the first set $U_{1}=30 \mathrm{~cm} / \mathrm{s}$ and in the second $U_{1}=50 \mathrm{~cm} / \mathrm{s}$, resulting in the local Reynolds numbers at the measurement station of approximately 7200 and 12000 respectively.

Since it was observed that in all these cases the mixed fluid p.d.f.'s were quite uniform across the width of the layer, the composition field of the whole layer, in each case, can be characterized by an average p.d.f. The evolution of the composition field is shown in figure 12 in terms of average p.d.f.'s, defined by

$$
\bar{p}(\xi)=\frac{1}{y_{1}-y_{2}} \int_{y_{2}}^{y_{1}} p(\xi, y) \mathrm{d} y .
$$

The locations $y_{1}$ and $y_{2}$ were chosen such that the central $63 \%$ of the visual width of the layer was included in the integration. The actual height of the free-stream delta functions do exceed the clipped value in this figure. 

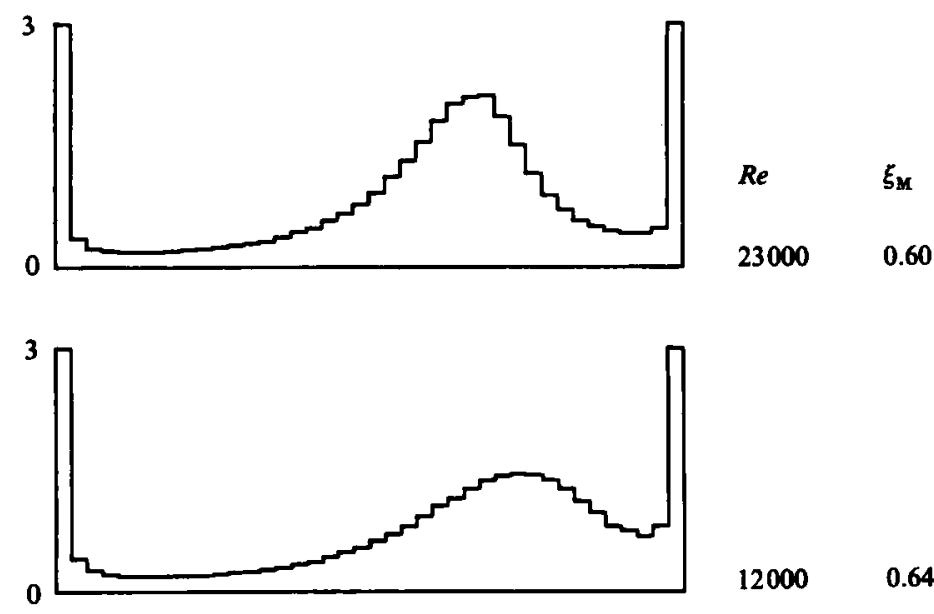

$12000 \quad 0.64$

$\bar{p}(\xi)$

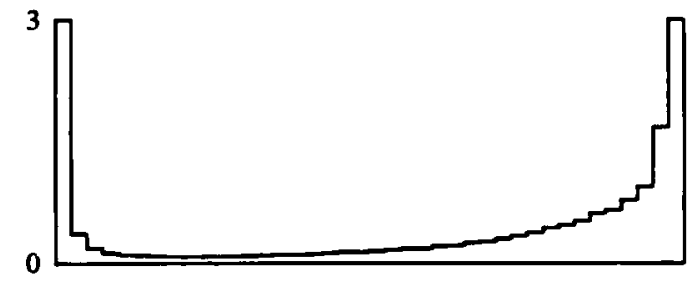

$7200 \quad 0.69$

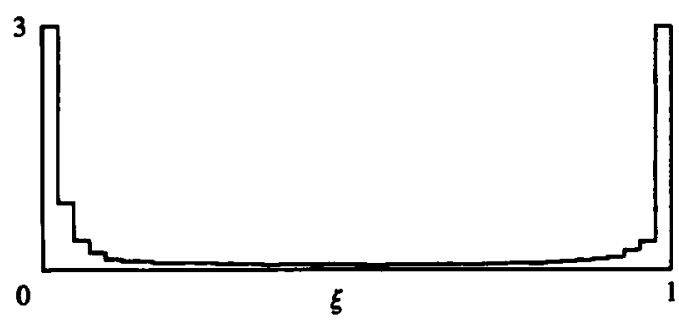

2800

FigURe 12. Evolution of the p.d.f. during the mixing transition.

For each of the four cases in figure 12 we also calculated from the detailed p.d.f.'s (not shown here) the total amount of product that would be formed in a simple one-step, fast and irreversible chemical reaction (Toor's (1962) analysis). In these calculations the average product concentration for a specific stoichiometric mixture fraction of the free-stream reactants $\xi_{\mathrm{s}}$ is computed by the integral

$$
\bar{C}_{\mathbf{p}}\left(y ; \xi_{\mathrm{s}}\right)=\int_{\epsilon}^{1-\epsilon} C_{\mathbf{p}}\left(\xi ; \xi_{\mathrm{s}}\right) p(\xi, y) \mathrm{d} \xi
$$

where $C_{\mathrm{p}}\left(\xi ; \xi_{\mathrm{s}}\right)$ is given by (4) (see figure 2) and $p(\xi, y)$ is the measured p.d.f. Note that the usual integration limits of 0 to 1 have been modified in order to be consistent with earlier definitions ( $(4)$ in handling the non-zero width of free-stream delta functions in the p.d.f. Calculations were made for the limiting cases of small and large $\xi_{s}$, for which we have

$$
\begin{array}{ll}
C_{\mathrm{p}}(\xi ; 1)=C_{10} \xi & \text { for } \xi_{\mathrm{s}} \rightarrow 1, \\
C_{\mathrm{p}}(\xi ; 0)=C_{20}(1-\xi) & \text { for } \xi_{\mathrm{s}} \rightarrow 0 .
\end{array}
$$




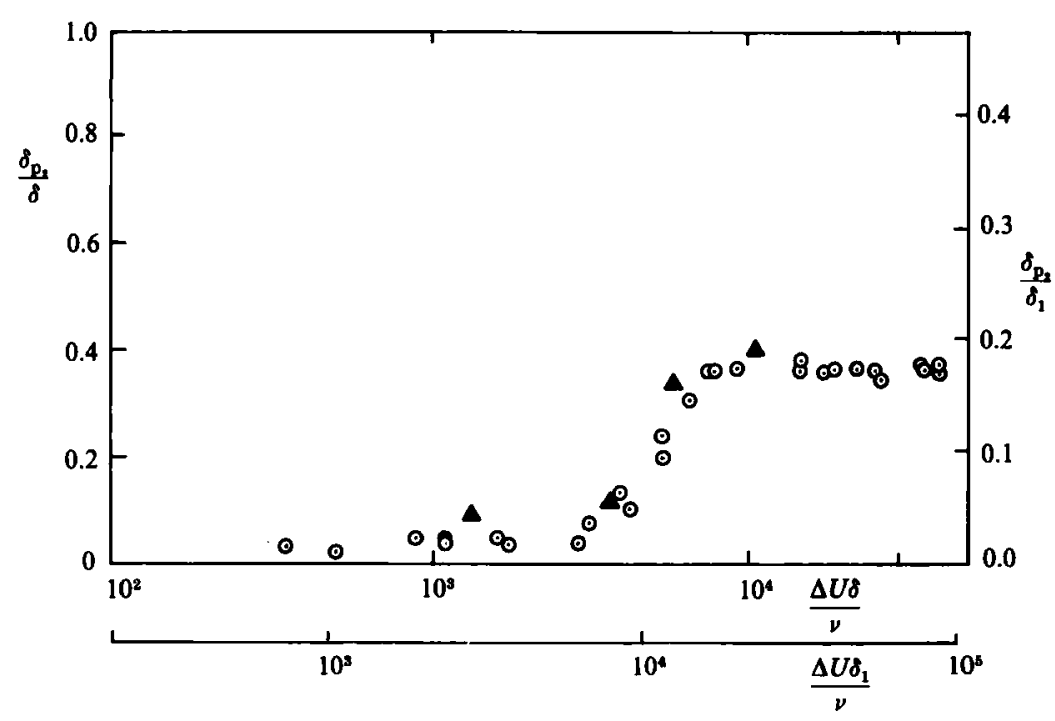

Figdre 13. Dependence on large-scale Reynolds number of the amount of product in a liquid mixing layer: $\odot$, Breidenthal; $\Delta$, calculated from present p.d.f.'s. $r=U_{2} / U_{1}=0.38$.

And finally, as a measure of the amount of product in the layer, the product thickness is used which, for the two limiting cases above, is defined by

$$
\delta_{\mathrm{p}_{1}}=\frac{1}{C_{10}} \int_{-\infty}^{+\infty} \bar{C}_{\mathrm{p}}(y ; 1) \mathrm{d} y, \quad \delta_{\mathrm{p}_{2}}=\frac{1}{C_{20}} \int_{-\infty}^{+\infty} \bar{C}_{\mathrm{p}}(y ; 0) \mathrm{d} y .
$$

These limiting product thicknesses are not in general equal due to the asymmetry of the mixed-fluid composition (see also Mungal \& Dimotakis 1984).

The amount of product in terms of the normalized product thickness, $\delta_{\mathbf{p}_{2}} / \delta_{1}$, is shown in figure 13 and is compared with the data of Breidenthal. In this figure, we have used a ratio of $\delta_{1}$ to the vorticity thickness $\delta$ of $\delta_{1} / \delta=2.1$, suggested by Brown \& Roshko (1974), to allow a comparison since Breidenthal presented his results using the vorticity thickness as a reference lengthscale.

The most important result of these measurements is that the composition of the mixed fluid changes continuously, in the mean, during the mixing transition (figure 12). In this region, not only does the amount of product change (figure 13), but also the product is found at a different range of compositions. At the beginning of the mixing transition, the fluid is found to be mixed mostly at very high values of concentration $\xi$. The distribution of mixed-fluid composition shifts toward lower values as mixing proceeds. Another way of looking at this evolution is to monitor the average mixed-fluid concentration of the entire layer $\xi_{M}$ which monotonically decreases during the transition (see the values in figure 12). The process seems to be one in which the newly entrained fluid continuously mixes with the existing fluid in the core in essentially a dilution process. Considering that the structure cores initially have a very large excess of high-speed fluid (see $\$ 5.1$ ), the continuous decrease of $\xi_{\mathrm{M}}$ and the shifting of the distribution toward lower values is qualitatively explained.

FiaURe 14. Evolution of the transverse profiles during the mixing transition: $\bigcirc, R e=7200 ; \triangle$, 12000; $\square, 23000$. (a) Pure high-speed fluid probability; $(b)$ pure low-speed fluid probability; $(c)$ total mixed-fluid probability; $(d)$ average mixed-fluid concentration. 

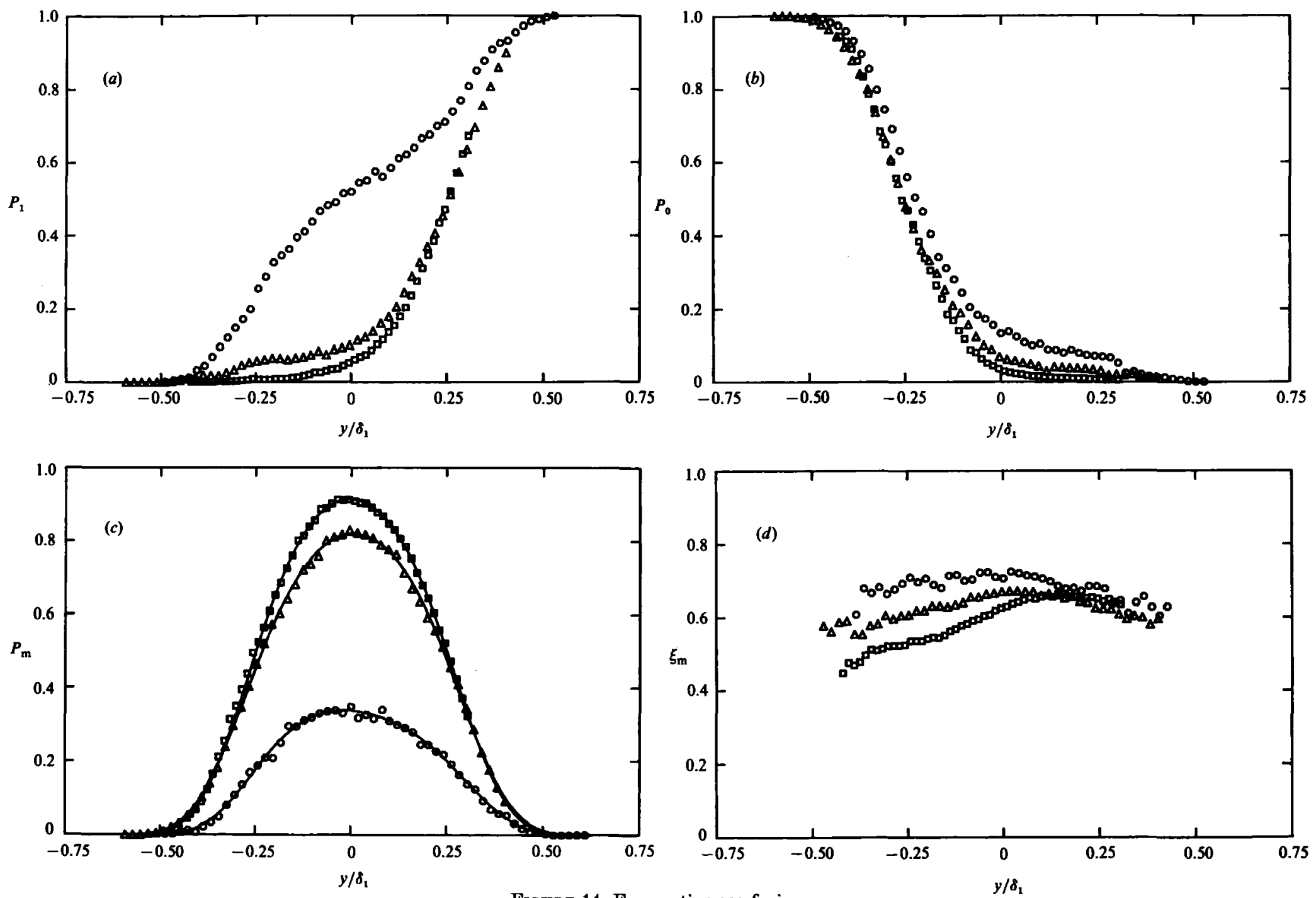

हू.

Figure 14. For caption see facing page. 
The concentration corresponding to the entrainment ratio $E$ is $\xi_{E}=E /(1+E)$, where $E$ is the volume ratio of the entrained high-speed to low-speed fluids. If the dilution mechanism proposed above is correct, $\xi_{M}$ should asymptote to $\xi_{E}$ sufficiently far downstream. Until this asymptotic state is reached, $\xi_{M}$ will necessarily be higher than $\xi_{E}$. In other words, the average composition in the mixing layer during the mixing transition is not determined only by the entrainment ratio, but also by the initial composition at roll-up. These results explain the previous observations made by Koochesfahani et al. (1985) of the very high mixed-fluid concentrations in a liquid mixing layer in the transition region. Similar observations in gas-phase layers have been made by Masutani (1985).

Figures $14(a-d)$ show the evolution of the transverse profiles calculated from the p.d.f.'s. Note that the initial large excess of pure high-speed fluid in the layer, still seen at $R e=7200$ in figure $14(a)$, very quickly diminishes as the introduction of three-dimensionality mixes the core fluid rapidly. Since there is originally a small amount of pure low-speed fluid in the cores, the changes in the probability of finding it $P_{0}$ are not as striking (figure $14 b$ ). The evolution of the total mixed-fluid probability $P_{\mathrm{m}}(y)$, figure $14(c)$, illustrates the increase of mixing during the transition. And finally the flatness of the $\xi_{\mathrm{m}}$ profile in figure $14(d)$ indicates that, even in the transition region, the mixed-fluid composition is quite uniform across the entire width of the layer. The shifting of $\xi_{m}$ to lower values is in accord with the evolution of the p.d.f.'s described previously.

It can be seen in figure 13 that the present estimates of the total amount of product from the measured p.d.f.'s are in good agreement with the results of Breidenthal. Owing to the resolution limitations discussed in $\$ 5.3$, however, these estimates can at best provide an upper limit to the amount of actual product that would be formed as the result of molecular mixing. Therefore it is believed that Breidenthal's value for the amount of product beyond the mixing transition may be too high.

\section{Chemical-reaction results}

The total amount of chemical product integrated across the layer was measured by Breidenthal (1981) in the limit of small stoichiometric mixture fraction $\xi_{\mathrm{s}}$. His results are shown in figure 13 in terms of the normalized product thickness $\delta_{\mathrm{p}_{2}} / \delta_{1}$. Owing to the asymmetry of the mixed-fluid composition, discussed in the previous section, a second different limit must also exist for very large $\xi_{\mathrm{s}}$ given by $\delta_{\mathrm{p}_{1}} / \delta_{1}$ (see $(14 a, b))$.

In this section we present chemical-reaction measurements, in the limit of both small and large stoichiometric mixture fractions, performed in the same highReynolds-number post-mixing-transition flow described in \$5.2. The motivation here is that these measurements, in addition to providing the mean product concentration profiles and the product thicknesses, would allow the determination of many quantities of interest in the limit of 'perfect' resolution. In particular, combining $(12),(13 a, b)$ and $(14 a, b)$ in $\$ 5.4$ with the definitions in $\$ 4$ results in the following relations:

$$
\begin{gathered}
P_{\mathrm{m}}(y)=\frac{\bar{C}_{\mathrm{p}}(y ; 1)}{C_{10}}+\frac{\bar{C}_{\mathrm{p}}(y ; 0)}{C_{20}}, \\
\xi_{\mathrm{m}}=\frac{\bar{C}_{\mathrm{p}}(y ; 1) / C_{10}}{\bar{C}_{\mathrm{p}}(y ; 1) / C_{10}+\bar{C}_{\mathrm{p}}(y ; 0) / C_{20}},
\end{gathered}
$$


(a)

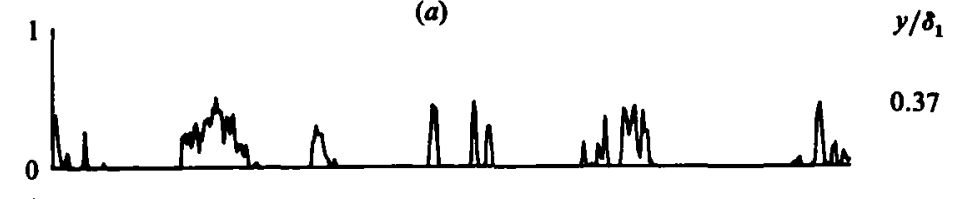

1

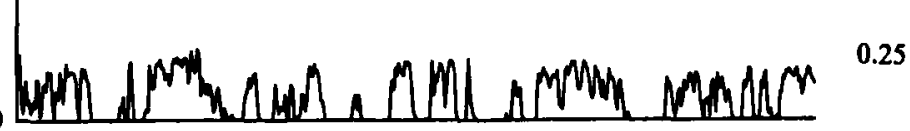

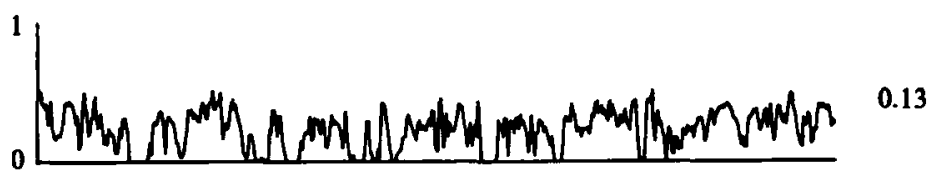

(b)

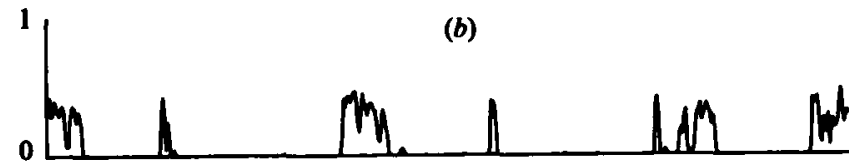

$y / \delta_{1}$

0.37

.

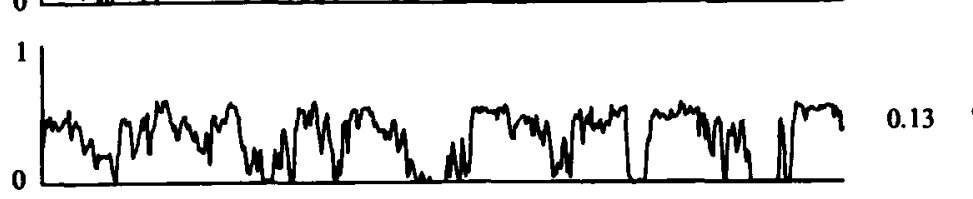

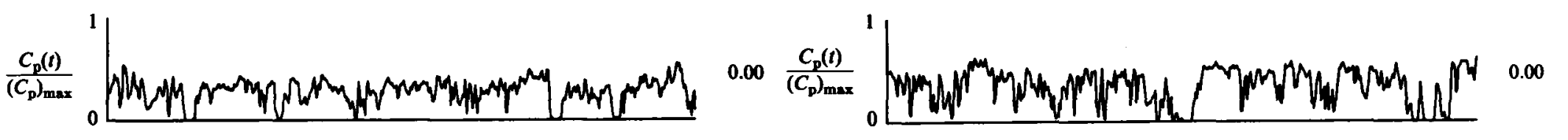
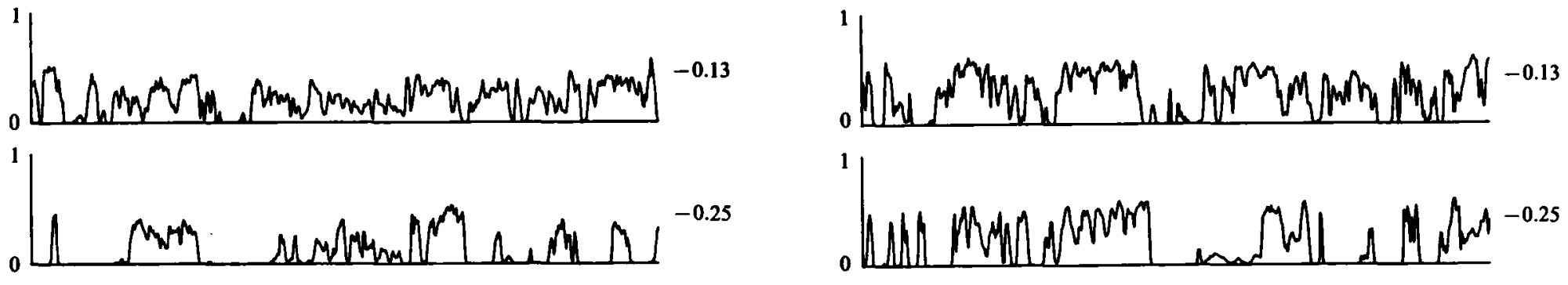

1

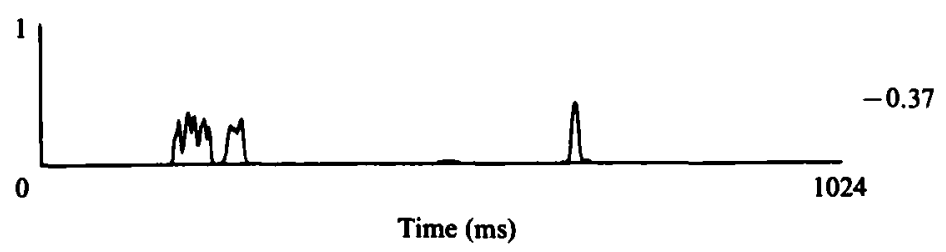

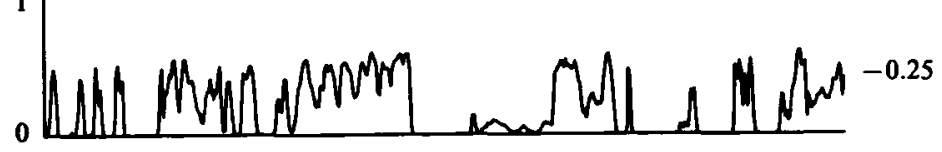
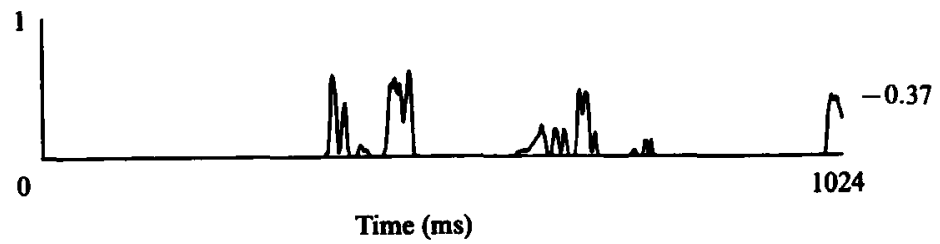

Time (ms)

우

Figure 15. Product concentration versus time, $r=0.38, R e=23000:(a) \xi_{\mathrm{s}}=0.09 ;(b) \xi_{\mathrm{s}}=0.91$. 


$$
\xi_{\mathrm{M}}=\frac{\delta_{\mathrm{p}_{1}}}{\delta_{\mathrm{p}_{1}}+\delta_{\mathrm{p}_{2}}}
$$

The limit of 'perfect' resolution is a consequence of the fact that the quantities mentioned above can be calculated from the average product-concentration profiles. As discussed in previous sections, the inability to resolve the smallest mixing scales does not affect the correct measurement of the mean concentration.

Two experiments were conducted at stoichiometric mixture fractions $\xi_{\mathrm{s}}=\mathbf{0 . 0 9}$ and 0.91 , which are believed to adequately represent the limits of $\xi_{s} \rightarrow 0$ and 1 . This is in fact a flip experiment with fixed acid/dye and base solutions, the calibration curve for which was presented in figure 1 . The variation of the product concentration with high-speed-fluid concentration $\xi$ for the two cases was shown in figure 3 . That the stoichiometric mixture fractions used here are adequate was confirmed by using the measured p.d.f.'s for this flow (figure 10) to estimate the amount of product according to the procedure described in $\$ 5.4$. It was found that at $\xi_{\mathrm{s}}$ of 0.09 and 0.91 the two product thicknesses had attained $95 \%$ of their asymptotic values. This is a reflection of the fact that there is very little mixed fluid to be found in the ranges $\xi<0.09$ and $\xi>0.91$.

It should be mentioned that the acid-base reaction used here is a diffusioncontrolled reaction. The Damkohler number for this experiment, defined by the ratio of the small-scale mixing to the chemical time, was estimated to be larger than 100 (see Koochesfahani 1984). The chemistry is therefore rapid compared to the relevant mixing times. Furthermore, the reaction results in negligible heat release and the flow is at uniform density.

\subsection{Flip experiment: $\xi_{\mathrm{s}}=0.09$ and 0.91}

These two experiments were preformed under flow conditions identical with those described in $\S 5.2$. Time traces of the product concentration, $C_{\mathrm{p}}(t)$, at selected points in the layer are shown in figures $(15 a, b)$. Each trace is composed of 512 consecutive data points connected by straight-line segments. The data are normalized by the maximum attainable product concentration $\left(C_{\mathrm{p}}\right)_{\max }$ (see $\S 2$ ). In order to see the underlying structure of the flow, the digital images of the whole layer corresponding to the data of figures $15(a, b)$ are displayed in figures $16(a, b)$. They were constructed from the time-series data at 150 points across the layer in the manner described in §5.1. The intensity assignment to the data is a linear variation between zero intensity at $C_{\mathrm{p}}=0$ and maximum intensity at $C_{\mathrm{p}}=\left(C_{\mathrm{p}}\right)_{\max }$. This is how the chemical product would look if it were being observed in real time during the experiment. The two horizontal lines mark the width of the layer $\delta_{1}$ (about $51.2 \mathrm{~mm}$ ).

Figures $15(a, b)$ show that the product concentration for $\xi_{\mathrm{s}}=0.91$ reaches higher values than for $\xi_{s}=0.09$. This can also be seen in the different intensities of the images in figure 16 which, in addition, show that it is possible to find instances when there is very little product anywhere across the layer. The average product concentration profiles for both cases are plotted in figure 17. Each profile consists of at least 150 points joined by straight-line segments. Note that the mean profiles appear quite symmetric and the peaks occur at the same position in the layer. The total amount of product for each case is found from the area under the average profile (see \$5.4). The final result is that the limiting product thicknesses in a liquid mixing layer above the mixing transition are given by

$$
\frac{\delta_{\mathrm{p}_{1}}}{\delta_{1}}=0.165 \text { for } \xi_{\mathrm{s}}=0.91, \quad \frac{\delta_{\mathrm{p}_{2}}}{\delta_{1}}=0.125 \text { for } \xi_{\mathrm{s}}=0.09
$$




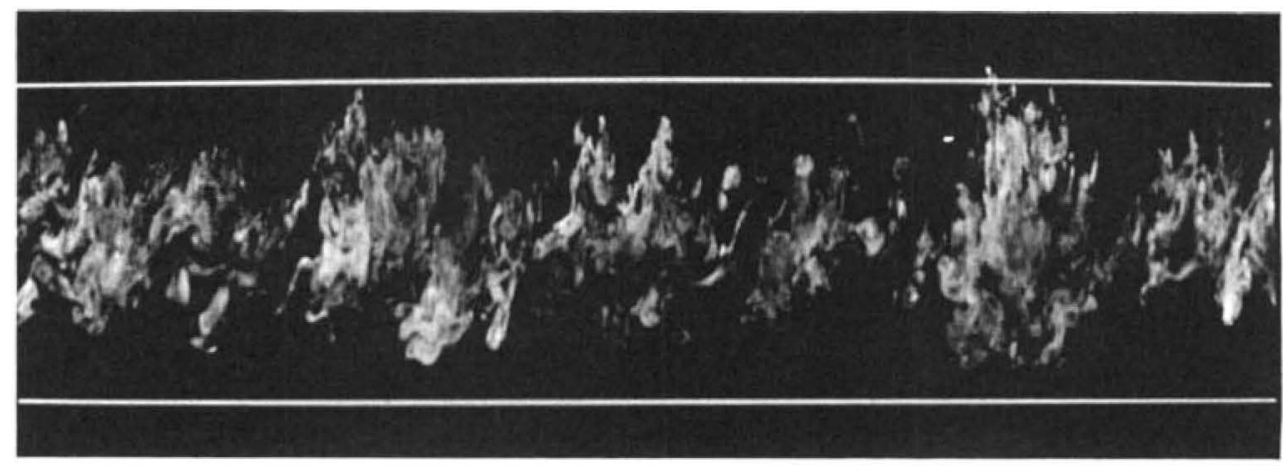

(a)

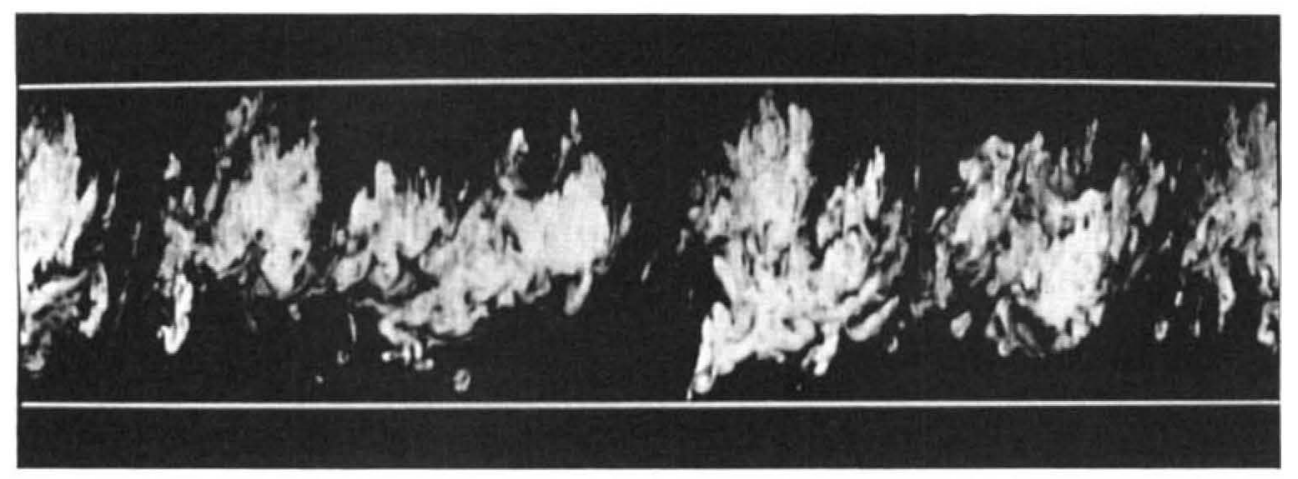

(b)

FiaURE 16. Digital LIF picture of the data of figure $15:(a) \xi_{\mathrm{s}}=0.09 ;(b) \xi_{\mathrm{s}}=0.91$.

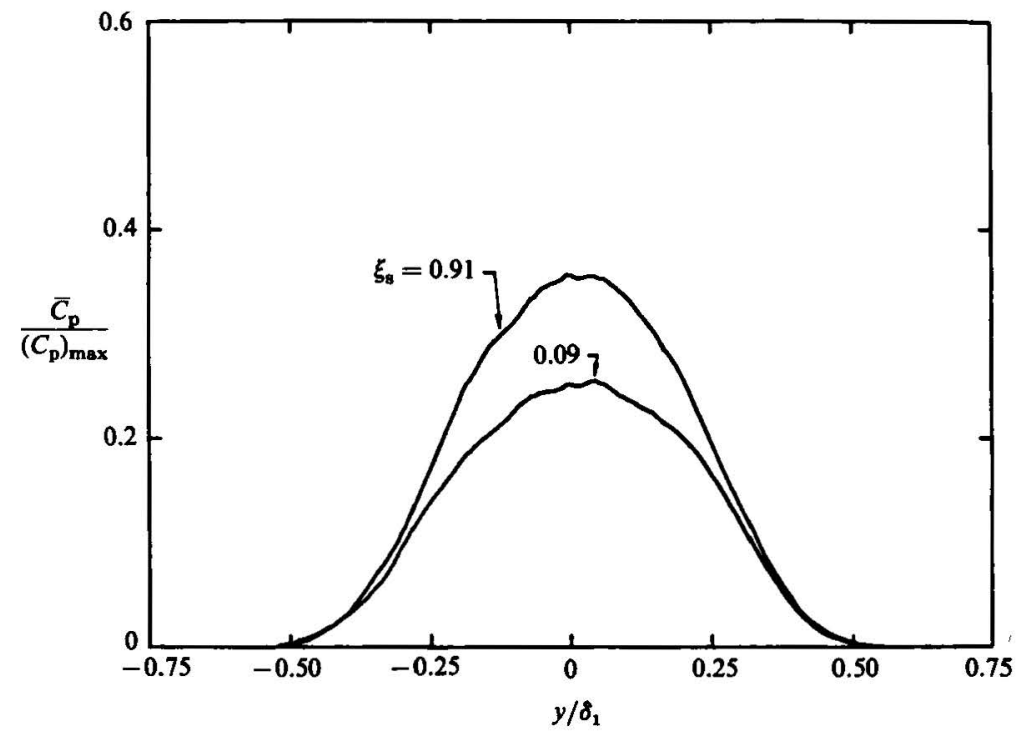

Figure 17. Average product concentration profiles: $R e=23000, r=0.38, \delta_{1}=51.2 \mathrm{~mm}$, area $=0.138\left(\xi_{\mathrm{s}}=0.09\right)$ and $0.182\left(\xi_{\mathrm{s}}=0.91\right)$. 
The $\xi_{\mathrm{s}}=0.91$ case has approximately $32 \%$ more product than that at $\xi_{\mathrm{s}}=0.09$. It is important to keep in mind that the only difference between the two cases discussed here is the exchange of side of the chemicals. That the amount of chemical product and the mean product profiles, in the same mixing layer, depend on which side of the layer the reactants are placed is a direct consequence of the asymmetry of the mixed-fluid composition in the layer.

The previous result of Breidenthal indicating a very weak dependence of the amount of product on Reynolds number (e.g. see figure 13) was confirmed by making a measurement at $\xi_{\mathrm{s}}=\mathbf{0 . 9 1}$ for a Reynolds number of 78300 (an increase by a factor of 3.4). The normalized product thickness $\delta_{\mathrm{p}_{1}} / \delta_{1}$ was found to be 0.167 . It should be noted that the estimate from the dilution p.d.f.'s sets the limiting value for $\xi_{\mathrm{s}} \rightarrow 0$ at 0.192 while Breidenthal's results give a value of 0.182 (see figure 13). The corresponding estimate from the p.d.f.'s for the $\xi_{s} \rightarrow 1$ limit gives a value of 0.289 for $\delta_{p_{1}} / \delta_{1}$. The overestimation of the amount of product is an inherent problem associated with the passive-scalar technique and has already been discussed in the previous section. The difference between the present result for $\xi_{s} \rightarrow 0$ and Breidenthal's value is believed to be due to some difficulties connected with the attentuation technique used by Breidenthal (see Koochesfahani 1984). The exact source of the discrepancy has not been firmly established however.

\subsection{Results in the limit of 'perfect' resolution}

The average product concentration profiles of figure 17 can now be used to get an estimate of various quantities in the limit of 'perfect' resolution according to (15)-(17). The first result is that the average mixed-fluid concentration for the entire layer $\xi_{M}$ is actually equal to 0.57 . This corresponds to a mixture of 1.32 parts of high-speed fluid to 1 part of low-speed fluid and agrees very well with the previously suggested value of entrainment ratio for this layer (Konrad 1976; Dimotakis 1984). Other results are presented in figures $18(a-c)$. In comparison with dilution results, the total probablity of finding mixed fluid $P_{\mathrm{m}}(y)$ in figure $18(a)$ shows the drawback of the passive scalar technique in determining the true extent of mixing. Note that the probability of finding unmixed fluid in the centre of the layer can be as high as 0.45 . This value is likely to be an upper limit since a small amount of mixed fluid whose composition is in the range for which the fluorescence is off has been excluded. An important finding is that the average concentration of mixed fluid $\xi_{\mathrm{m}}(y)$ is basically invariant across the width of the layer and is fixed at a value corresponding to the entrainment ratio. Therefore, a good portion of the lateral variation seen in the estimates from the dilution data is an artifact; a consequence of the resolution inadequacy of the measurement. It may also be noted from figure $18(b)$ that the agreement between the true $\xi_{\mathrm{m}}(y)$ profile and those estimated from dilution data generally improves as the low-speed side is approached. This is partly due to the non-uniformity of the effective resolution across the layer and its improvement as the low-speed side is approached as a result of lower local flow speed. It is believed that this is also why the estimates of $\xi_{M}$ based on dilution data tend to be higher than the actual values.

In addition to the quantities presented so far, the probabilities of finding unmixed fluid from low- and high-speed sides, $P_{0}$ and $P_{1}$ respectively, can also be determined in the limit of 'perfect' resolution. 'This is done by manipulating the relations given in $\S 4$ to show that

$$
\bar{\xi}=P_{1}+\xi_{\mathrm{m}}(y) P_{\mathrm{m}}(y)
$$



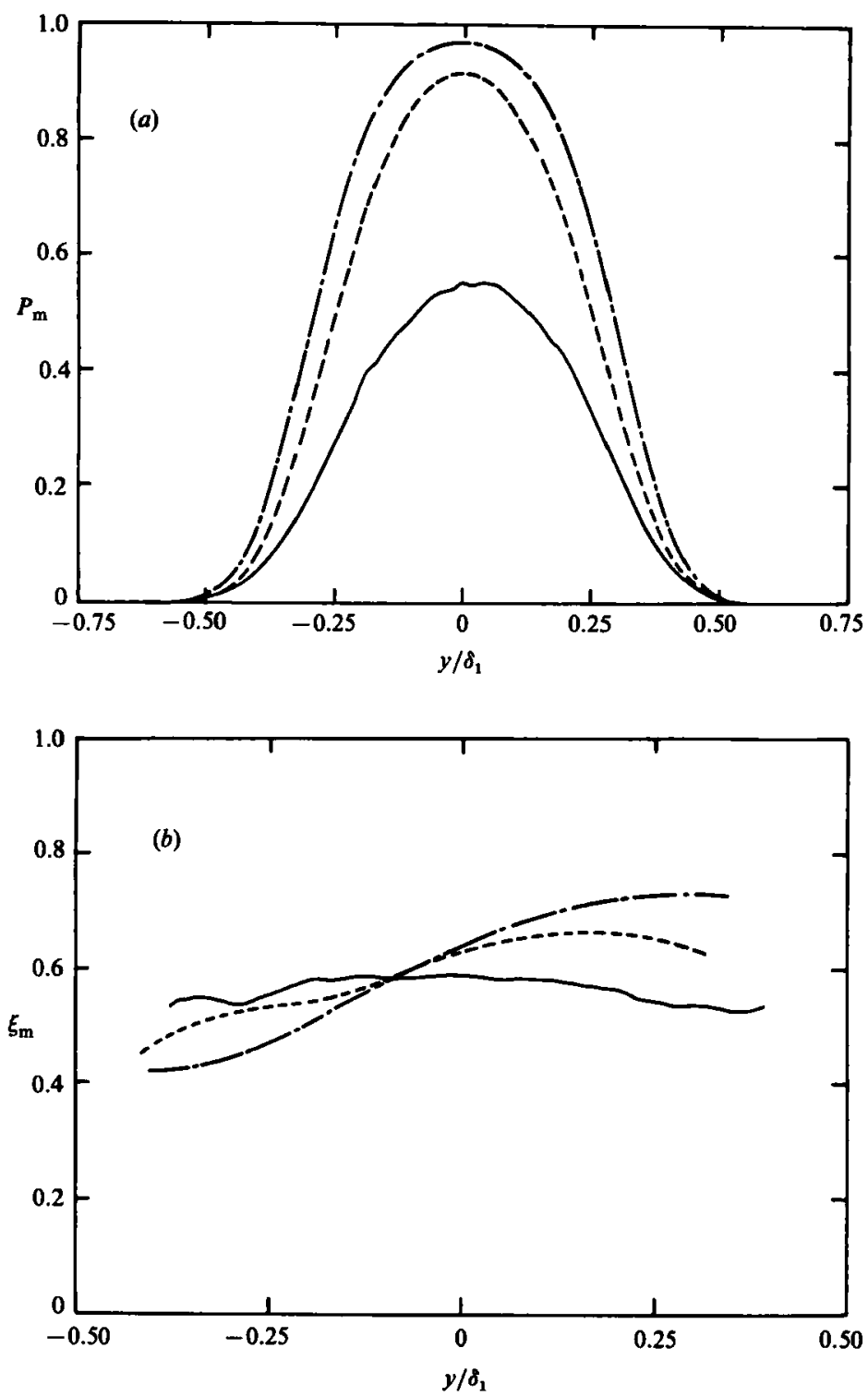

Figurg $18 a, b$. For caption see page 106.

and noting that the $\bar{\xi}$ profile, figure $11(a)$, does not suffer from resolution problems. $P_{0}$ is then readily found using the normalization condition

$$
P_{0}(y)+P_{\mathrm{m}}(y)+P_{1}(y)=1 .
$$

The profiles are shown in figure $18(c)$ compared with dilution results. It is interesting to see that the $P_{1}$ profile tends to have a somewhat flat region in the middle of the layer. This may be a remnant of the initial large excess of high-speed fluid in the core at the time of roll-up (see §5.1).

It has already been mentioned that, once above the mixing transition, increasing the Reynolds number by a factor of 3.4 left the limiting value of $\delta_{\mathrm{p}_{1}} / \delta_{1}$ virtually 


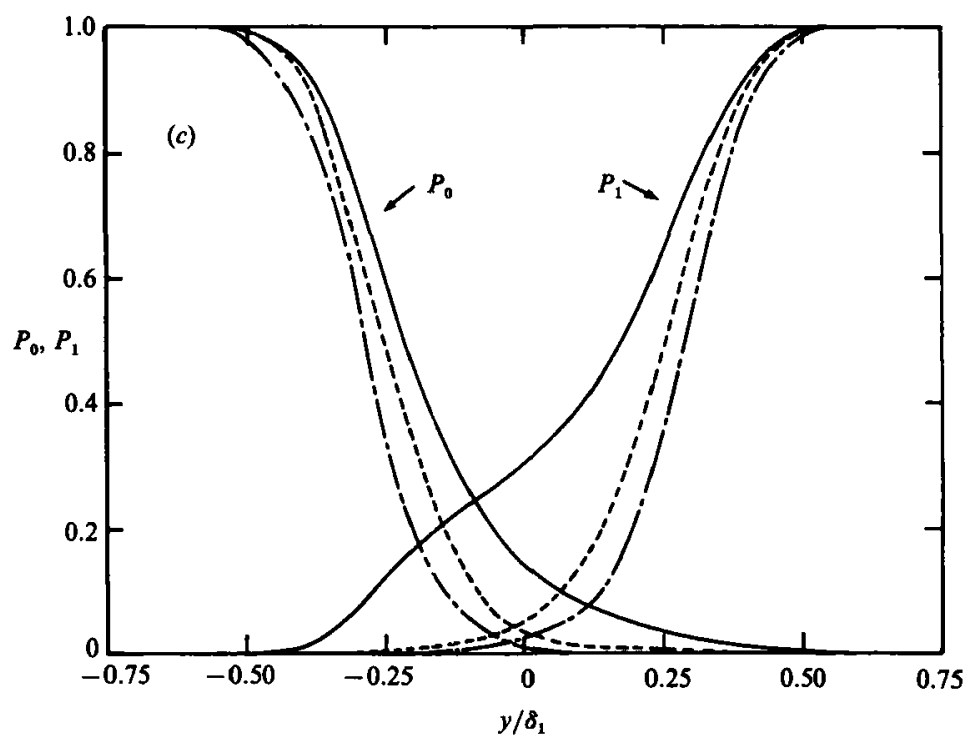

Figure 18. Transverse profiles in the limit of 'perfect' resolution: - , 'perfect' resolution; ---, dilution (0.8 ms scan); ---, dilution ( $8.0 \mathrm{~ms}$ scan). (a) Total mixed-fluid probability ; $(b)$ average mixed-fluid concentration; (c) pure high- and low-speed fluid probabilities.

unchanged. Results of Breidenthal had previously indicated that in the other limit $\delta_{\mathrm{p}_{2}} / \delta_{1}$ is also independent of Reynolds number. Therefore, according to (17), the average mixed-fluid concentration of the layer $\xi_{M}$ necessarily becomes fixed at 0.57 above the mixing transition. If the fluids that get entrained into the layer become mixed with the existing mixed fluid some time later, the constancy of $\xi_{M}$ implies that an asymptotic state has been reached with a fixed entrainment ratio $E$ such that $\xi_{E}=E /(1+E)=\xi_{M}$. Here $\xi_{E}$ is the concentration of fluid mixed at the entrainment ratio. Thus, according to this argument, $\xi_{E}=0.57$ or $E=1.32$. Note also that, in this case, $E=\delta_{\mathrm{p}_{1}} / \delta_{\mathrm{p}_{2}}$ so that the two limiting product thicknesses would become equal only if the entrainment ratio were equal to unity.

\section{Comparisons}

Comparisons are presented here with chemical-reaction results of Mungal \& Dimotakis (1984) and passive-scalar measurements of Konrad (1976), both of which were performed in gas-phase mixing layers.

\subsection{Amount of product}

It is known from the work of Konrad (1976), Breidenthal (1981) and Mungal \& Dimotakis (1984) that gases and liquids produce different amounts of product. Mungal \& Dimotakis have reported the limiting values in the gas-phase layer to be $\delta_{\mathrm{p}_{1}} / \delta_{1}=0.264$ and $\delta_{\mathrm{p}_{2}} / \delta_{1}=0.237$. Results by Breidenthal and Mungal \& Dimotakis had previously indicated that the amount of product in the gas-phase layer was $20 \%$ to $25 \%$ more than in a liquid. The present data, however, support a larger difference. The gas-phase case, as represented by the data of Mungal \& Dimotakis, has approximately twice the amount of product of the liquid layer investigated here. We 
note that this difference in the amount of product in gases and liquids (i.e. fluids of differing Schmidt number) is not accounted for in traditional models of turbulent transport and mixing.

In the model of Broadwell \& Breidenthal (1982), the total amount of product in the layer is divided into a homogeneous part and a flame-sheet part. The relative amounts contributed by each part must be determined from experiment. According to our results, the homogeneous region and the flame sheets contribute equally to the total product in a gas-phase layer. It should be mentioned that the amount of product in gas-phase layers is a weak function of Reynolds number and decreases by $20 \%$ for a factor of 10 increase in Reynolds number as reported by Mungal, Hermanson \& Dimotakis (1985). The amount of product in a liquid layer has a much weaker dependence on Reynolds number as evidenced by experimental results and also suggested by the Broadwell-Breidenthal model. Thus, strictly speaking, comparisons between liquids and gases should be made with this restriction in mind.

The validity of the comparison between the liquid- and gaseous-product measurements, described above, requires some discussion. One issue is that, in the present liquid experiments, a measurement at a downstream station $x=x_{1}$ gives the amount of product formed as a result of the local mixture composition at that station. In the gas-phase experiment of Mungal \& Dimotakis, by contrast, the amount of product measured at $x_{1}$ is the cumulative amount of product formed between the splitter plate $(x=0)$ and $x_{1}$, a consequence of the irreversibility of the chemical reaction in that experiment. Thus comparing the results of these two types of experiments may not be valid in general. This would be the case when there is a substantial minimum activation energy required for the reaction to proceed, for example, or if the chemistry is slow. In the special case of fast chemistry and spontaneous reactions (i.e. hypergolic reactants) the comparison is valid. The reason is that, in this case, no more product can be formed than that dictated by the reactant in short supply (i.e. the lean reactant). Therefore, the total amount of product at $x_{1}$ would be the same whether the reactants were to react, irreversibly, starting at $x=0$, or to mix passively, without any reaction, and then instantaneously react to completion at $x_{1}$.

Another factor in the comparison is that, in the present experiment, an amount of product corresponding to the range of mixture ratios for which the fluorescence is off is missing from the total product. This contribution, however, was shown to be small for the choice of stoichiometric mixture fractions used in these experiments.

\subsection{Distribution of composition}

The average concentration of mixed fluid $\xi_{\mathrm{m}}(y)$ in the liquid layer, measured in the limit of 'perfect' resolution, was presented in figure $18(b)$. We are not aware of any equivalent results for the gas-phase case. A comparison is presented, nevertheless, in figure 19 using the data available from Konrad and Mungal \& Dimotakis. Konrad's measurement of this quantity is based on a passive scalar technique and, therefore, is likely to be suffering from resolution problems. The technique of using a pair of flip experiments at high and low stoichiometric mixture fractions to estimate $\xi_{m}$ in the limit of 'perfect' resolution can certainly be also applied to the gas-phase case. Assuming that the mean product profiles of Mungal \& Dimotakis, at $\xi_{\mathrm{s}}=0.89$ and 0.11 , are adequate for this purpose, we estimated the $\xi_{\mathrm{m}}$ profile for the gas case using (16) in the same manner as for the liquid case. Figure 19 indicates that the mean mixed-fluid concentration in a gas-phase layer varies considerably more across the 


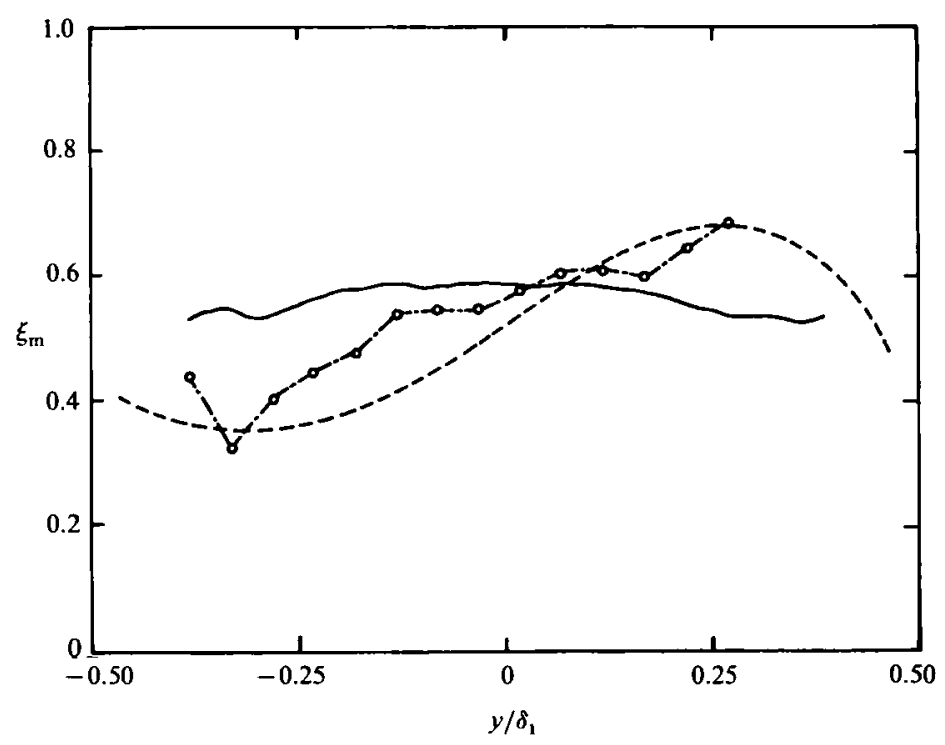

Figure 19. Comparison of the average mixed-fluid concentration: - , present (liquid); ---, Mungal \& Dimotakis (1984) (gas); O---O, Konrad (1976) (gas).

layer than its liquid counterpart. This implies that the distribution of values of the composition of the mixed fluid in a gas-phase shear layer must be a stronger function of the transverse coordinate $(y)$ than for a liquid-phase layer.

We can also use the mean product profiles of Mungal \& Dimotakis to obtain an estimate of the total mixed-fluid probability $P_{\mathrm{m}}(y)$ in the gas-phase layer (equation (15)). The estimate, not shown here, produces a bell-shaped curve quite similar to that for a liquid, see figure $18(a)$, but with a peak approaching the value of unity. Similar curves have been obtained in the passive scalar measurements of Fiedler (1974) and Konrad (1976) with peaks as high as 0.95. Comparing liquid-and gas-phase $P_{\mathrm{m}}(y)$ curves at comparable Reynolds number shows that the gas case has roughly twice the amount of mixed fluid as its liquid counterpart, consistent with the difference in the amount of chemical product in these two cases discussed earlier.

The p.d.f.'s for liquid and gas mixing layers at the same velocity ratio and comparable Reynolds number are shown in figure 20. Also shown is the result of recent theoretical calculations by Kollmann \& Janicka (1982), which are similar to the p.d.f.'s calculated by Pope (1981). Even though a passive scalar technique is used in the measurement of the p.d.f.'s in both cases, and therefore they may be contaminated due to lack of resolution, some general conclusions can be drawn. It can be seen that the overall structure of the p.d.f. of the mixed-fluid composition in a gas is quite similar to a liquid in that there exists a predominant composition which is asymmetric and the same at all points across the layer. This is to be contrasted with the calculated results which, for example, predict that the peak of the distribution marches as the layer is traversed. The p.d.f.'s indicate that in the gas there is more mixed fluid of high concentration $\xi$ on the high-speed side and more of low $\xi$ toward the low-speed side, compared to the liquid. It is emphasized that it is this behaviour which is most likely to be responsible for the lateral variation of the $\xi_{\mathrm{m}}$ profile in the gas and not the shift in the position of the peak.

It should be mentioned that the p.d.f.'s measured by Batt (1977) (for a $r=0$ gas-phase shear layer) appear to agree with the theoretical calculations mentioned 


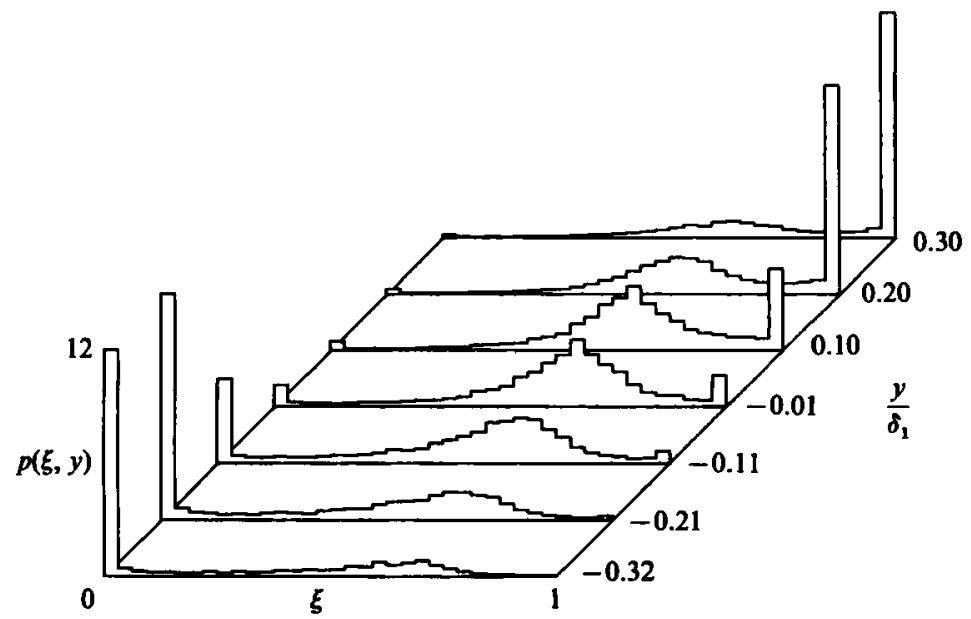

(a)

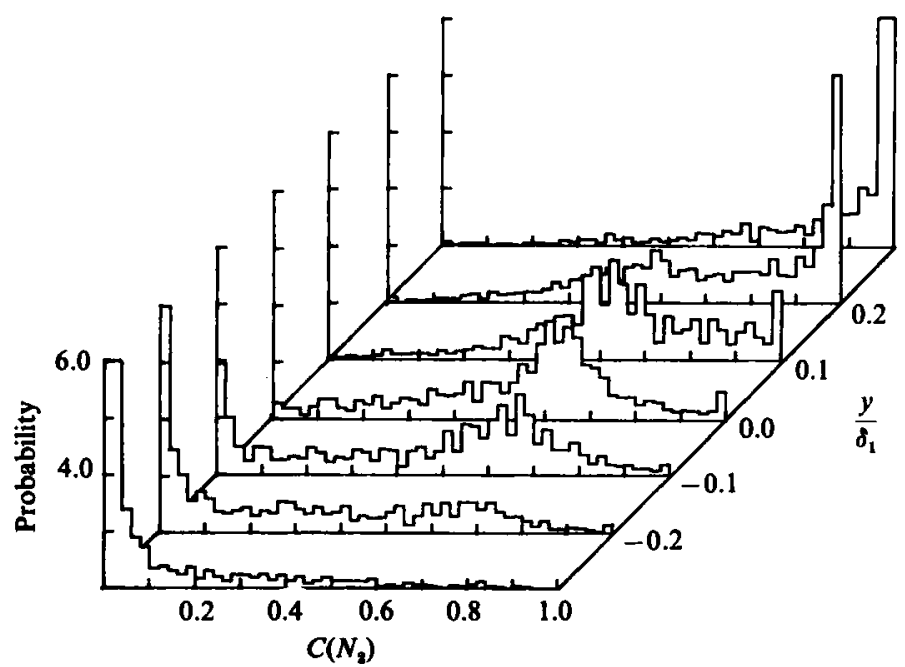

(b)

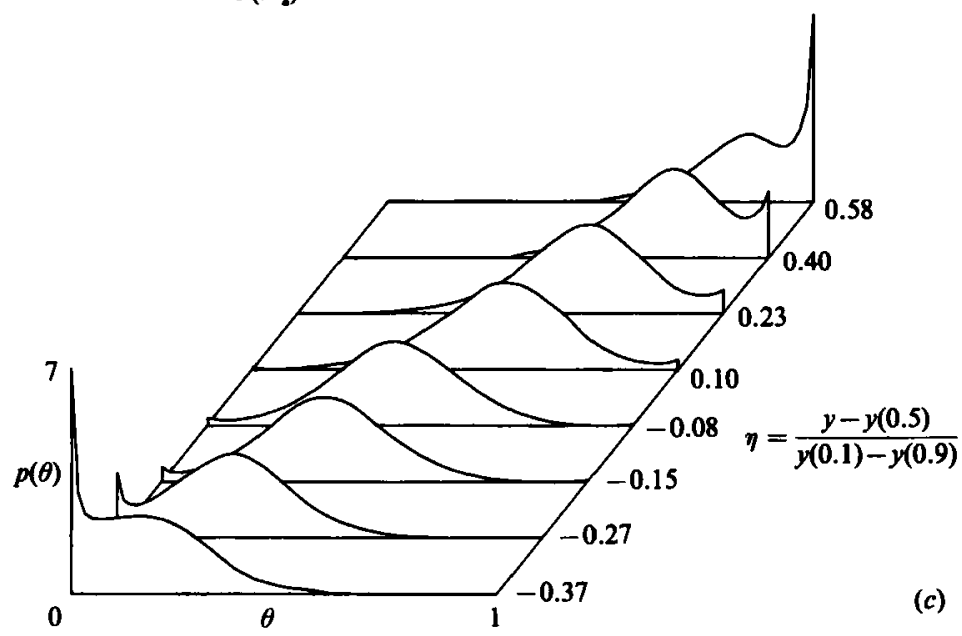

Figure 20. Comparison of the p.d.f.'s: (a) present (liquid), (b) Konrad (1976) (gas), (c) Kollmann \& Janicka (1982) (calculations). 


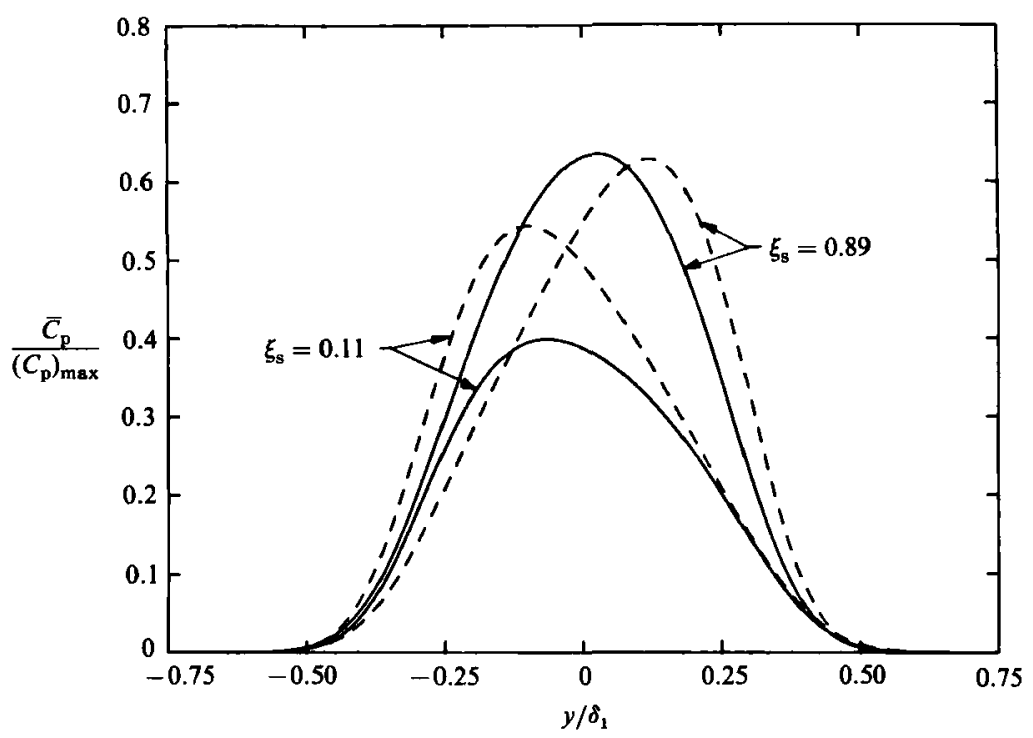

Figure 21. Comparison of average product concentration profiles:----, Mungal \& Dimotakis (1984) (gas); - , calculated from present p.d.f.'s (liquid).

above and do not share the features of Konrad's results. It has been pointed out by Mungal \& Dimotakis (1984) that Batt's experimental resolution, which is about two orders of magnitude worse than Konrad's, may be the cause of the discrepancy between the two p.d.f.'s. Supporting evidence for this conjecture can also be found in the trend in the mean mixed-fluid concentration, figure $11(d)$, for the data obtained with the deliberately degraded measurement resolution.

From (16) it is clear that the $\xi_{\mathrm{m}}$ profile is very useful in determining the behaviour of the average product profiles in the limit of large and small stoichiometric mixture fractions. In particular, we have

$$
\frac{\bar{C}_{\mathrm{p}}(y ; 1)}{\bar{C}_{\mathrm{p}}(y ; 0)} \sim \frac{\xi_{\mathrm{m}}(y)}{1-\xi_{\mathrm{m}}(y)} .
$$

Now it is clear that the average product profiles for the liquid at $\xi_{\mathrm{s}}$ of 0.09 and 0.91 , figure 17, are about the same, within a scale factor, since the mean mixed-fluid concentration varies very little across the layer. Changes in the mean product profiles and the shift in their peaks in a flip experiment can be interpreted to be a consequence of the non-uniformity of the $\xi_{\mathrm{m}}$ profile. Therefore, based on figure 19 , we expect the mean product profiles to change and their peaks to shift more in a flip experiment performed in a gas layer than in a liquid. This is illustrated in figure 21 . The curves for the liquid were calculated from the measured p.d.f.'s (figure 10) since direct measurements of product at the same stoichiometric mixture fractions as those in the gas were not available. The small shift of the peak in the calculated liquid curves is an artifact of the apparent variation of the $\xi_{\mathrm{m}}$ profile found from dilution data.

\section{Conclusions}

The results presented show that the vortical structures in the mixing layer initially roll-up with a large excess of high-speed fluid in the cores. Closely related to this, it is found that the mean composition of the mixed fluid during the mixing transition 
is not determined solely by the entrainment ratio but also by the initial composition at roll-up. During the mixing transmition, not only does the amount of mixed fluid increase, but its composition also changes. At the beginning of the mixing transition, the fluid is mostly found to be mixed at very high values of concentration and, accordingly, the p.d.f. is considerably asymmetric in favour of the high-speed side. The distribution of mixed fluid shifts toward lower concentrations as mixing proceeds. It is proposed that a dilution mechanism, in which the newly entrained fluid continuously mixes with the existing fluid in the cores, may explain this behaviour.

The p.d.f. of the mixed fluid, above the mixing transition and also throughout the mixing transition, is uniform across the entire transverse extent of the layer. It is shown that the consequences of the measurement-resolution inadequacies encountered in the commonly used passive scalar technique can be severe. A technique is described which, by employing a flip experiment, allows the total amount of mixed fluid and its average, among other quantities, to be determined in the limit of 'perfect' resolution. Results in this limit reveal that the probability of finding unmixed fluid in the centre of the turbulent liquid mixing layer, above the mixing transition, could be as high as $\mathbf{0 . 4 5}$. This should be compared with the value in the range of 0 to 0.06 that has been reported for gas-phase layers. In addition, the average concentration of mixed fluid across the layer is found to be essentially at a fixed value corresponding to the entrainment ratio.

Results indicate that the mixed-fluid composition is more uniform across a liquid shear layer than in the gas-phase layer. Direct measurements of the amount of chemical product show that the liquid layer, at high Reynolds number, has approximately $50 \%$ less product than that reported by Mungel \& Dimotakis (1984) in the gas-phase case. This implies that molecular diffusion (Schmidt number) does play a role in turbulent mixing of high Reynolds number flows. The amount of chemical product, in the same mixing layer, depends on which side of the layer the reactants are placed (corresponding to a flip experiment at stoichiometric mixture fractions of $\xi_{\mathrm{s}}$ and $1-\xi_{\mathrm{s}}$ ). This is a direct consequence of the asymmetry of the mixed-fluid composition resulting from the asymmetric entrainment into the layer.

We would like to acknowledge the valuable contributions of Drs D. B. Lang and F. A. Roberts during the course of this research. The many illuminating discussions with Drs R. E. Breidenthal, J.E. Broadwell and M. G. Mungal are very much appreciated. This work was sponsored by the Air Force Office of Scientific Research Contract Nos F44620-76-C-0046, F49620-79-C-0159 and Grant No. AFOSR-83-0213.

\section{REFERENCES}

Batchelor, G. K. 1959 Small-scale variation of convected quantities like temperature in turbulent fluid. Part 1. General discussion and the case of small conductivity. J. Fluid Mech. 5, 113-133.

BAtт, R. G. 1977 Turbulent mixing of passive and chemically reacting species in a low-speed shear layer. J. Fluid Mech. 82, 53-95.

Bernal, L. P. 1981 The coherent structure of turbulent mixing layers: I. Similarity of the primary vortex structure. II. Secondary streamwise vortex structure. Ph.D. thesis, Caltech.

Bernal, L. P., Breidenthal, R. E., Brown, G. L., Konrad, J. H. \& Roshko, A. 1979 On the development of three-dimensional small scales in turbulent mixing layers. In Turbulent shear flows 2: Second Intl Symp. on Turbulent Shear Flows, Imperial College London, 2-4 July, pp. 305-313. Springer. 
BILGER, R. W. 1980 Turbulent flows with nonpremixed reactants. In Turbulent reacting flows (ed. P. A. Libby \& F. A. Williams), Topics in Applied Physics, vol. 44, pp. 65-113. Springer.

Breidenthal, R. E. 1981 Structure in turbulent mixing layers and wakes using a chemical reaction. J. Fluid Mech. 109, 1-24.

Broadwell, J. E. \& Breidenthal, R. E. 1982 A simple model of mixing and chemical reaction in a turbulent shear layer. J. Fluid Mech. 125, 397-410.

Brown, G. L. \& Roshko, A. 1971 The effect of density differences on the turbulent mixing layer. In Turbulent shear flows, AGARD-CP-93, 23.1-23.12.

Brown, G. L. \& Roshko, A. 1974 On density effects and large structure in turbulent mixing layers. J. Fluid Mech. 64, 775-816.

Dimotakis, P. E. 1984 Two-dimensional shear layer entrainment. AIAA-84-0368.

Effelsberg, E. \& Peters, N. 1983 A composite model for the conserved scalar PDF. Combust. Flame 50, 351-360.

Fiedler, H. E. 1974 Transport of heat across a plane turbulent mixing layer. Ad. Geophy. 18A, 93-109.

GANJi, A. R. \& SaWYER, R. F. 1980 Experimental study of the flowfield of a two-dimensional premixed turbulent flame. AIAA J. 18, 817-824.

Jones, W. P. \& Whitela W, J. H. 1982 Calculation methods for reacting turbulent flows: A review. Combust. Flame 48, 1-26.

Kollmann, W. \& Janicka, J. 1982 The probability density function of a passive scalar in turbulent shear flows. Phys. Fluids, 25, 1755-1769.

KonRAD, J. H. 1976 An experimental investigation of mixing in two-dimensional turbulent shear flows with applications to diffusion-limited chemical reactions. Ph.D. thesis, Caltech; and Project SQUID Tech. Rep. CIT-8-PU.

Koochesfahani, M. M. 1984 Experiments on turbulent mixing and chemical reactions in a liquid mixing layer. Ph.D. thesis, Caltech.

Koochesfahani, M. M., Dimotakis, P. E. \& Broadwell, J. E. 1985 A 'flip' experiment in a chemically reacting turbulent mixing layer. $A I A A J .23,1191-1194$.

Koochesfahani, M. M. \& Dimotakis, P. E. 1985 Laser induced fluorescence measurements of mixed fluid concentration in a liquid plane shear layer. AIAA J. 23, 1700-1707.

Masutani, S. M. 1985 An experimental investigation of mixing and chemical reaction in a plane mixing layer. Ph.D. thesis, Stanford University, HTGL Topical Rep. No. T-246.

Mungal, M. G. \& Dimotakis, P. E. 1984 Mixing and combustion with low heat release in a turbulent shear layer. J. Fluid Mech. 148, 349-382.

Mungal, M. G., Hermanson, J. C. \& Dimotakis, P. E. 1985 Reynolds number effects on mixing and combustion in a reacting shear layer. $A I A A J .23,1418-1423$.

Pitz, R. W. \& DaIty, J.W. 1983 Combustion in a turbulent mixing layer formed at a rearward-facing step. AIAA J. 21, 1565-1570.

Pope, S. B. 1981 A Monte Carlo method for the PDF equations of turbulent reactive flow. Combust. Sci. Technol. 25, 159-174.

Srefivivasan, K. R., Tavoularis, S. \& Corrsin, S. 1981 A test of gradient transport and its generalizations. In Turbulent shear flows 3: Third Intl Symp. on Turbulent Shear Flows, The University of California, Davis, 9-11 September, pp. 96-112. Springer.

Toor, H. L. 1962 Mass transfer in dilute turbulent and nonturbulent systems with rapid irreversible reactions and equal diffusivities. AIChE J. 8, 70-78.

WALK ER, B. J. 1979 Turbulence model comparisons for shear layers and axisymmetric jets. $U . S$. Army Missile Command Tech. Rep. RD-80-1.

WaLlace, A. K. 1981 Experimental investigation of the effects of chemical heat release in a turbulent shear flow. Ph.D. thesis, University of Adelaide, Australia, AFOSR-TR-84-0650.

Winant, C. D. \& Browand, F. K. 1974 Vortex pairing: the mechanism of turbulent mixing-layer growth at moderate Reynolds number. J. Fluid Mech. 63, 237-255. 